\title{
Cart3D Simulations for the Second AIAA Sonic Boom Prediction Workshop
}

\author{
George R. Anderson* \\ Science and Technology Corporation, Moffett Field, CA 94035 \\ Michael J. Aftosmis ${ }^{\dagger}$ and Marian Nemec ${ }^{\ddagger}$ \\ NASA Ames Research Center, Moffett Field, CA 94035
}

\begin{abstract}
Simulation results are presented for all test cases prescribed in the Second AIAA Sonic Boom Prediction Workshop. For each of the four nearfield test cases, we compute pressure signatures at specified distances and off-track angles, using an inviscid, embedded-boundary Cartesian-mesh flow solver with output-based mesh adaptation. The cases range in complexity from an axisymmetric body to a full low-boom aircraft configuration with a powered nacelle. For efficiency, boom carpets are decomposed into sets of independent meshes and computed in parallel. This also facilitates the use of more effective meshing strategies each off-track angle is computed on a mesh with good azimuthal alignment, higher aspect ratio cells, and more tailored adaptation. The nearfield signatures generally exhibit good convergence with mesh refinement. We introduce a local error estimation procedure to highlight regions of the signatures most sensitive to mesh refinement. Results are also presented for the two propagation test cases, which investigate the effects of atmopsheric profiles on ground noise. Propagation is handled with an augmented Burgers' equation method (NASA's sBOOM), and ground noise metrics are computed with LCASB.
\end{abstract}

\section{Nomenclature}

\begin{tabular}{|c|c|c|c|}
\hline$A_{\text {ref }}$ & Reference area & $\Phi$ & Off-track/Azimuthal angle \\
\hline$C_{D / L / M}$ & Drag/lift/pitching moment coefficients & \multirow{2}{*}{\multicolumn{2}{|c|}{ Subscripts }} \\
\hline & Local pressure coefficient & & \\
\hline$e$ & Integrated signature differences & $(\cdot)_{\infty}$ & Freestream value \\
\hline$E$ & Local error estimate & $(\cdot)_{t}$ & Stagnation value \\
\hline $\mathcal{J}$ & Aerodynamic output functional & $(\cdot)_{\mathrm{c}}$ & Coarse \\
\hline$\ell$ & Distance along signature & $(\cdot)_{\mathrm{f}}$ & Fine \\
\hline$L$ & Reference length for propagation & $(\cdot)_{\mathrm{m}}$ & Medium \\
\hline$M$ & Mach number & \multirow{2}{*}{\multicolumn{2}{|c|}{ Abbreviations }} \\
\hline$p$ & Static pressure & & \\
\hline $\mathrm{p}$ & Order of convergence & \multicolumn{2}{|c|}{ ASEL/CSEL A-/C-weighted sound exposure level } \\
\hline$r$ & Distance from flight path & AXIE & Axisymmetric body (Case I) \\
\hline$T$ & Temperature & \multicolumn{2}{|c|}{ AXIE-PROP Axisymmetric body (Prop. Case I) } \\
\hline$w$ & Weight in functional & $\mathrm{C} 25 \mathrm{~F}$ & C25D with flow-through nacelle (Case III) \\
\hline$\alpha$ & Angle of attack & $\mathrm{C} 25 \mathrm{P}$ & C25D with powered nacelle (Case IV) \\
\hline$\beta$ & $\sqrt{M_{\infty}^{2}-1}$ & JWB & JAXA wing-body (Case II) \\
\hline$\theta$ & Offset angle to avoid sonic glitch & LCASB & Loudness Code for Asymmetric Sonic Booms \\
\hline$\mu$ & Mach angle $=\sin ^{-1}\left(1 / M_{\infty}\right)$ & LM-1021 & Lockheed Martin 1021 (Prop. Case II) \\
\hline$\rho$ & Density & $\mathrm{PL}$ & Perceived level of noise \\
\hline$\tau$ & Normalized $x$-distance from nose Mach cone & SBPW & Sonic Boom Prediction Workshop \\
\hline
\end{tabular}

*Aerospace Engineer, Computational Aerosciences Branch. george.anderson@nasa.gov. Member AIAA.

$\dagger$ Aerospace Engineer, Computational Aerosciences Branch. michael.aftosmis@nasa.gov. Associate Fellow AIAA.

$\ddagger$ Aerospace Engineer, Computational Aerosciences Branch. marian.nemec@nasa.gov. Member AIAA. 


\section{Introduction}

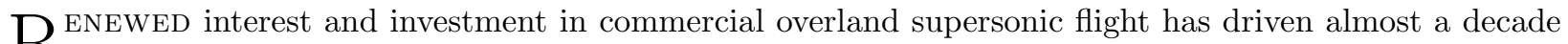
$\left\{\begin{array}{l}\text { of steady improvement in numerical simulation methods for predicting, characterizing, and reducing } \\ \text { ond }\end{array}\right.$ the objectionable sonic booms generated by such aircraft. In support of this effort, three boom prediction workshops have been organized:

2008: NASA Fundamental Aeronautics Program Sonic Boom Prediction Workshop ${ }^{1}$

2014: AiAA Sonic Boom Prediction Workshop 1 (SBPW1) ${ }^{2}$

2017: AiAa Sonic Boom Prediction Workshop 2 (sBPw2) ${ }^{3}$

The purpose of these workshops is both to document the evolving state of the art in boom prediction methods and provide focus for future investment and research efforts.

Like its predecessors, SBPW2 includes a set of nearfield benchmark simulations. It also introduces a new, complementary set of atmospheric propagation benchmarks, which specifically exercise the techniques used for propagation of the near-aircraft pressure signal to the ground and computation of noise metrics. Participants are provided with a common set of inputs (i.e. geometries, grids, flight conditions) but otherwise apply their own simulation tools and best practices to compute the requested outputs.

Reflecting a general convergence of boom prediction methodologies, the workshop is decoupled into nearfield and propagation components, as depicted in Figure 1. "Nearfield" indicates the region near the aircraft, where the flow field exhibits significant three-dimensional effects, driven by the details of the vehicle geometry. To capture these effects, simulations of the nearfield region (usually within several body lengths of the vehicle) are typically performed with computational fluid dynamics (CFD) codes used for aircraft aerodynamic analysis. Farther from the body, a quasi-one-dimensional approximation becomes appropriate, and the effects of atmospheric variability and absorption become critical. This region is usually simulated with more specialized propagation codes, which incorporate wind and temperature profiles and account for classical and relaxation losses. As an exercise, workshop participants are encouraged to attempt a complete aircraft-to-boom simulation, by applying a pipeline of simulation tools to compute a nearfield signature, propagate it to the ground, and compute the ground noise. However, because this tends to obscure the uncertainties

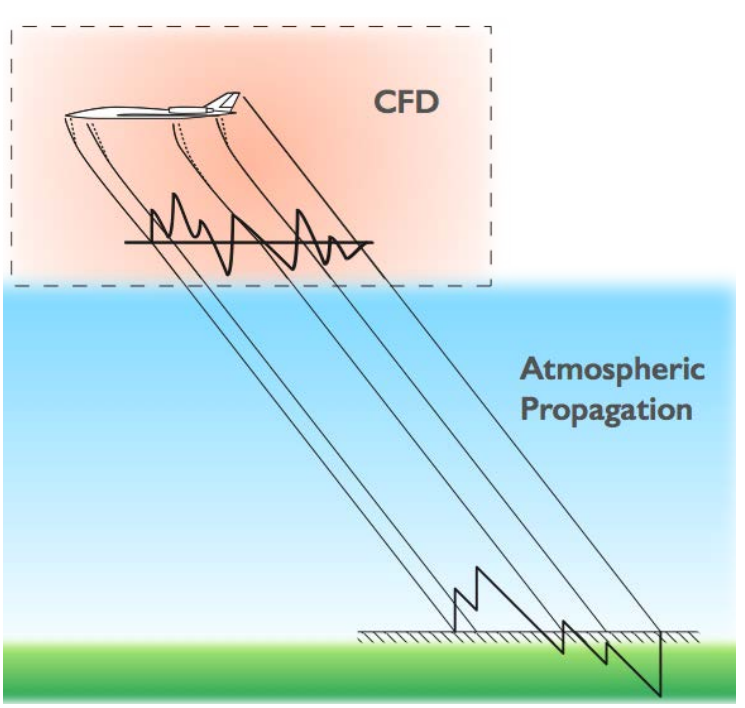

Figure 1: Decomposition of boom prediction into nearfield CFD and atmospheric propagation inherent in each component, the formal nearfield and propagation halves of the workshop are kept independent.

This paper presents results submitted to SBPW2 for all four nearfield cases. The vehicles for the nearfield portion are shown in Figure 2. For all cases, the Mach number is 1.6 and the altitude is $15.76 \mathrm{~km}$. By design, all four designs generate broadly similar on-track signatures. Depending on the vehicle design and wind conditions, off-track booms (located laterally away from the flight path) can easily be louder than the on-track boom. ${ }^{a}$ Recognizing this, SBPW2 requires computation of boom "carpets" — pressure signatures for a range of off-track angles. Results are also presented for both propagation cases, which are based on vehicles used in both SBPW1 and SBPW2. These cases investigate the impact of atmospheric profiles on propagation.

Section II discusses our approach to boom prediction in detail, focusing on refinements to previous work. ${ }^{4-6}$ Notably, we discuss mesh rotation techniques to improve azimuthal mesh alignment and investigate the efficacy of decomposing the carpet into multiple independent meshes computed in parallel. Adjoint-based mesh adaptation is leveraged throughout the work, and we also introduce a technique to localize discretization error estimates along each signature using a simple Richardson extrapolation approach. After presenting and discussing the nearfield and propagation results in Sections III and IV, we conclude with some general remarks on boom prediction with Cartesian methods.

${ }^{\text {a }}$ Indeed this proves to be true for all the nearfield cases considered in this workshop. 


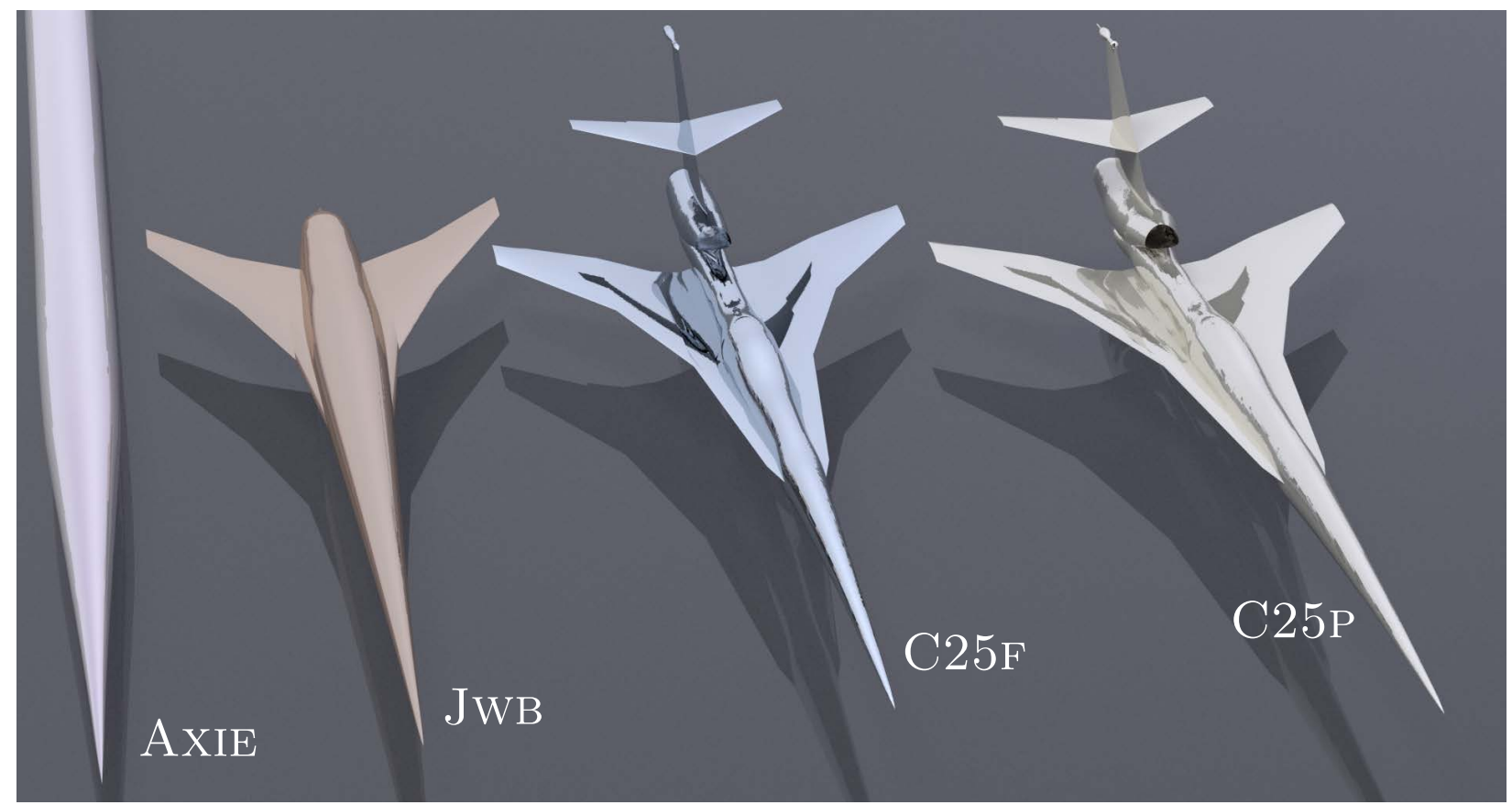

Figure 2: Models for the nearfield portion of the Second AIAA Sonic Boom Prediction Workshop. From left to right: I. AxIE, an axisymmetric body; II. JwB, the JAXA wing-body; III. C25F, the NASA Concept 25D model with flow-through nacelle; IV. C25P, Concept 25D with powered engine.

\section{Approach and Numerical Methods}

By convention, we use the cylindrical coordinate frame illustrated in Figure 3, with the origin located at the vehicle nose. The streamwise coordinate, $x$, is aligned with the nominal flight path, and runs aft from $x=0$ at the nose. $x$ is roughly the distance along the vehicle's longitudinal axis, except that the angle of attack $\alpha$ is built into the geometry. $r$ denotes the radial distance from the $x$-axis, and is often normalized by a reference length $L$. The cylindrical coordinate frame's azimuthal angle $\Phi$ is conventionally called the "off-track" angle, with "on-track" indicating $\Phi=0^{\circ}$, i.e. directly below the flight path. It is convenient to express distances along each sensor in normalized form: ${ }^{7}$

$$
\tau=\frac{1}{L}(x-r \beta)
$$

with $\beta=\sqrt{M_{\infty}^{2}-1}$. Thus $\tau=0$ lies on the Mach cone originating from the geometry nose (assumed to be at the origin), with half-angle $\mu=\sin ^{-1}\left(1 / M_{\infty}\right)$. At one reference length $L$ further along the sensor, $\tau=1$.

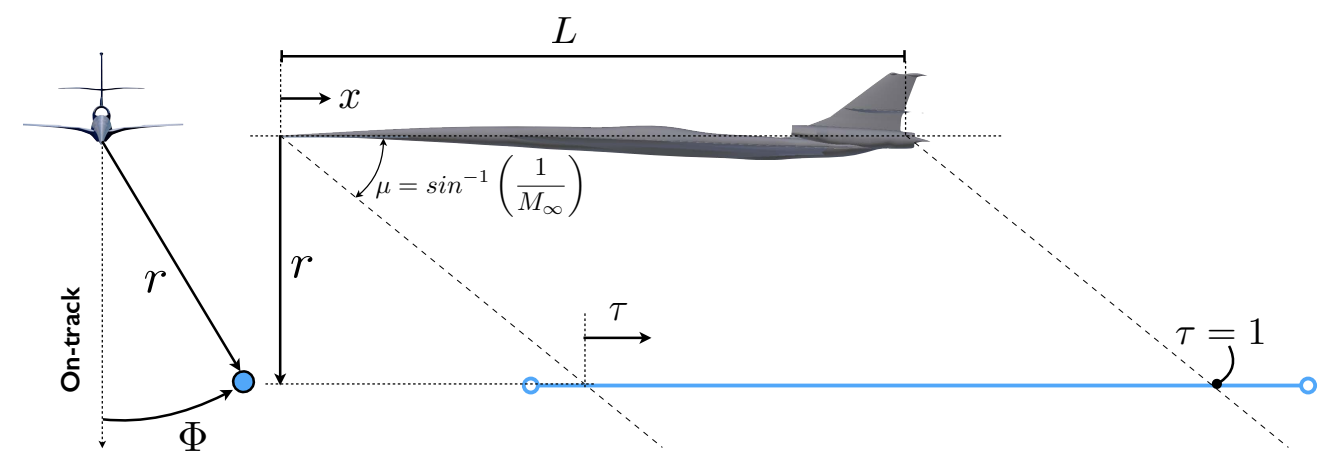

Figure 3: Coordinate frame and notation used for defining sensor locations 


\section{II.A. Nearfield Approach}

The near-aircraft simulations in this paper are computed with the Cart3D simulation package. ${ }^{8}$ The flow solver is a second-order, finite-volume code, in which the Euler equations are discretized on multilevel Cartesian meshes with embedded "cutcell" boundaries. Meshing is automatic, and adjoint-based adaptation is used to efficiently evaluate the off-body pressure signatures without using an excessive number of cells. The flow and adjoint solvers are parallel and highly scalable. ${ }^{9}$ For this work, we use the flux-vector splitting approach of van Leer ${ }^{10}$ and the low-dissipation Barth-Jespersen limiter. ${ }^{11}$ This method has been used extensively for sonic boom prediction and for low-boom design optimization, ${ }^{4-6,12}$ and more detail is available in previous publications. ${ }^{8,13,14}$

\section{Parallel Decomposition of Boom Carpet}

In previous work, problems involving multiple off-track angles have typically been solved by combining all sensors into a single mesh. ${ }^{5,6}$ This is advantageous when there is large overlap in the regions where the sensors demand high mesh resolution. While this is the case for sensors at different radial distances, far less mesh refinement is common among sensors at widely-spaced off-track angles. Experimentation in SBPW1 showed that splitting a $90^{\circ}$-carpet among two meshes proved far more accurate than using a single Cartesian mesh. ${ }^{6}$ Likewise, in preliminary experimentation with the SBPW2 cases, it proved extremely challenging and prohibitively expensive to accurately compute all six off-track angles with balanced accuracy in a single mesh.

In this work we make extensive use of carpet partitioning. As illustrated in Figure 4, we split each six-azimuth boom carpet problem into six independent meshes. Decomposing the carpet in this manner makes the problem becomes embarrassingly parallel - each off-track angle can be computed independently. Another advantage is that each adjoint-based mesh adaptation problem is focused on fewer outputs. Since the sensors compete less for mesh refinement, the signature quality stays more balanced without the need for heuristic adaptation functional weighting. ${ }^{5}$ However, what proved most efficacious about splitting is that it permits the use of two techniques that greatly improve meshing efficiency:

1. Azimuthal mesh alignment: Each mesh can be aligned to its off-track angle $\Phi$, which reduces numerical dissipation.

2. Stretching: The aligned meshes can be stretched to

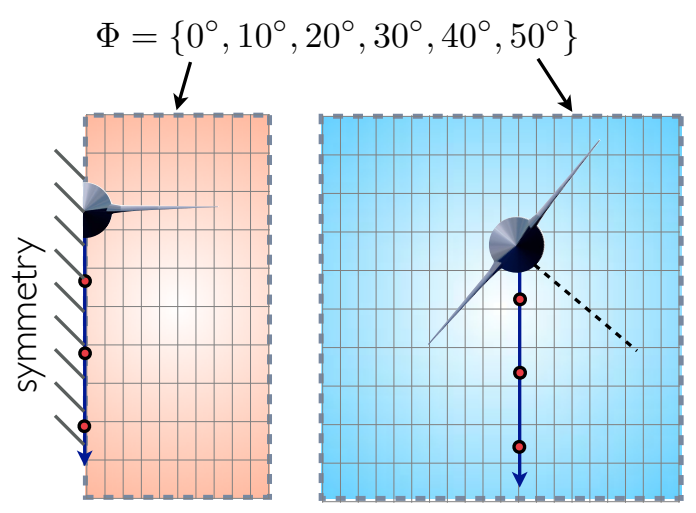

Figure 4: Nearfield boom carpet decomposition and azimuthal mesh alignment for computing offtrack signatures much higher aspect ratios.

Each of these is discussed in the following sections. Note that without splitting, we found these techniques to have limited applicability. Conversely, without leveraging them, the mesh splitting approach alone is much less advantageous. When all used together, however, they substantially accelerate the process over analysis with a single Cartesian mesh.

We also experimented with grouping in sets of two or three off-track angles per mesh. Ultimately, our studies showed that with all the efficiency improvements described in the following sections, it was most efficient to fully partition the SBPW2 cases into six separate meshes. Note that this result is partly a function of the relatively wide angular spacing, $\Delta \Phi=10^{\circ}$, between consecutive azimuths in the discretized boom carpet. If the spacing had been, for example, $\Delta \Phi=1^{\circ}$, there would be more overlap in mesh refinement, and we would expect clustering to be appropriate.

\section{Mesh Alignment with Propagation}

As also observed with many other CFD approaches, ${ }^{1,15}$ early investigations showed that Cartesian meshes can be highly effective at computing off-body signatures, provided that the mesh is nearly aligned with the Mach-angle $\mu$ of the freestream flow. ${ }^{4}$ Good Mach alignment reduces dissipation and permits the mesh to be stretched in the propagation direction, further reducing the cell count. ${ }^{4}$ Together, this easily allows computation of on-track signatures at distances of $r / L=5$ or more. ${ }^{5}$ 
However, perfect alignment with the Mach angle leads to a face-normal Mach number of unity, which is susceptible to the "sonic glitch" phenomenon. ${ }^{16}$ As in previous work, this is avoided by aligning the mesh to $\mu \pm \theta$, where $\theta \ll \mu .^{4}$ In this work, we found that $0.25^{\circ}<\theta<0.5^{\circ}$ best avoids the sonic glitch without introducing excessive dissipation. The choice of adding or subtracting $\theta$ impacts the behavior at shocks each sign will induce either small over-shoots or under-shoots at pressure peaks, and the opposite at troughs.

This mesh alignment technique promotes propagation of signals from the geometry to on-track sensors. ${ }^{5,6}$ In previous work with Cartesian meshes, off-track signatures have generally been computed with meshes aligned only with the Mach wave. ${ }^{5,6}$ In SBPw1, off-track angles closer to $\Phi=90^{\circ}$ than to $\Phi=0^{\circ}$ were shown to be more efficiently handled by "yawing" the mesh to the Mach cone at $\Phi=90^{\circ}{ }^{6}$ Both approaches are misaligned for important intermediate off-track angles, such as $\Phi=20^{\circ}-50^{\circ}$. For this workshop, experimentation with the AXIE case (where the axial symmetry dictates that signatures are identical at all $\Phi$ ) revealed that the signature quality severely degrades for azimuthal misalignment of greater than $10^{\circ}$ without resorting to large cell counts. ${ }^{b}$

Therefore, for this workshop, we make extensive use of azimuthal mesh rotation in addition to the standard Mach angle mesh rotation. As illustrated in Figure 4, to align the mesh with off-track propagation, the mesh is "rolled" about the streamwise $x$-axis. Thereafter, the mesh is rotated to the Mach angle in this frame. This can be expressed as a two-step transformation of a coordinate in the original frame:

$$
\left[\begin{array}{l}
x \\
y \\
z
\end{array}\right]_{\text {mesh }}=\underbrace{\left[\begin{array}{ccc}
\cos (\mu \pm \theta) & 0 & -\sin (\mu \pm \theta) \\
0 & 1 & 0 \\
\sin (\mu \pm \theta) & 0 & \cos (\mu \pm \theta)
\end{array}\right]}_{\text {Mach angle }} \underbrace{\left[\begin{array}{ccc}
1 & 0 & 0 \\
0 & \cos \Phi & -\sin \Phi \\
0 & \sin \Phi & \cos \Phi
\end{array}\right]}_{\text {Azimuth }}\left[\begin{array}{l}
x \\
y \\
z
\end{array}\right]_{\infty}
$$

The second transformation matrix (applied first) rolls the frame to align it with the off-track signature, and then the first matrix applies the usual Mach angle rotation. Some of the signs depend on the direction of rotation - in practice we keep the mesh frame fixed and use Equation (2) to rotate the geometry and sensor descriptions. This is analogous to wind tunnel tests, where off-track signatures are measured by rolling the geometry. When needed, the transformation can be inverted to return certain outputs to the freestream frame.

One seeming disadvantage of azimuthal rotation is the loss of the ability to use a symmetry condition for all but $\Phi=0^{\circ}$. However, adaptation to off-track sensors focuses most of the mesh resolution in one half plane anyway. Our studies show that the gains from good alignment far outweighed the relatively small penalty from losing the symmetry condition.

While our approach requires that we generate multiple meshes, and rotate each twice, the process is easily automated. The domain is set such that it contains the entire domain of influence of the geometry on the sensors. This is computed automatically by intersecting the Mach cone opening backward from the nose with a set of Mach cones opening forward from the endpoint of each sensor.

\section{Mesh Aspect Ratio}

Previous work has demonstrated that stretching the mesh to an aspect ratio of 2-4 in all but the Mach wave normal direction greatly improves the cost/accuracy ratio. ${ }^{4}$ After extensive experimentation for sBPw2, we determined that an even larger aspect ratio of 8 was most effective for all cases and azimuths, with two caveats. First, the sensors must be strictly azimuthally aligned to be "on-track" in the rotated computational domain, as described above - any unaligned signatures will degrade faster. Second, the accuracy of aerodynamic coefficients appears to gradually worsen with increasing aspect ratio. For more general purposes, such high aspect ratios may therefore be inappropriate. For the workshop, however, we found that they significantly reduced the cell counts required for a given signature accuracy.

\section{Adjoint-Based Mesh Adaptation}

We use an output-based mesh adaptation method based on the method of adjoint-weighted residuals. ${ }^{17}$ The mesh adaptation is targeted to reduce the discretization error in a scalar functional $\mathcal{J}$, which combines all the relevant outputs of interest. For the workshop, the primary outputs are the off-body pressure signatures

\footnotetext{
${ }^{\mathrm{b}} \mathrm{A}$ similar study in earlier work found that somewhat larger azimuths are feasible, but for a far less complex signature, generated by a SEEB-like geometry. ${ }^{5}$
} 
located at specified distances and azimuths from the body. At each extraction location, we add a term comprised of a weighted integration of squared-pressure along the sensor:

$$
\mathcal{J}_{r}=\int_{0}^{L} w(\ell)\left(\frac{p(\ell)-p_{\infty}}{p_{\infty}}\right)^{2} d \ell
$$

The local weight profile along the sensor, $w(\ell)$, allows different segments of the signature to be emphasized or de-emphasized. All sensor functionals are then linearly combined into a scalar adaptation functional:

$$
\mathcal{J}=\sum_{r} w_{r} \mathcal{J}_{r}
$$

Although a significant portion of the mesh refinement required by closer sensors overlaps with regions also needed for farther sensors (which is why it is worthwhile to compute them with a single mesh), this overlap is not complete. To accurately resolve signatures at closer distances, they must appear explicitly in the adaptation functional. Note that because we split the off-track angles into separate meshes, there is no need for azimuthal weighting.

Our investigations indicate that the computed signatures are not excessively sensitive to the adaptation weights $w(\ell)$ and $w_{r}$. In many cases, however, custom weighting can be beneficial, although it is challenging to do so in a principled manner. The specific weights used for each case in SBPW2 are based on experience, not on formal optimality, but we use a few principles, which are summarized below.

Weights can serve at least three distinct purposes. One is to balance the relative magnitude of each term. Due to the form of Equation (3), regions with a locally greater magnitude of $p-p_{\infty}$ contribute more to the functional. Figure 5 shows how the natural $1 / \sqrt{r}$ decay in pressure magnitude greatly reduces the contribution of farther off-body sensors to the total. To counteract this, we typically set $w_{r}$ proportionally higher for farther sensors. Similarly, near the front of each signature, the thin nose generates very small pressure variations, which leads to those regions being naturally de-emphasized in the functional. To help capture the initial pressure rise we often apply larger weights $w(\ell)$ near the beginning of each sensor.

A second purpose of weights is to assign a relative "importance" to each sensor and each location along the sensor. For example, for the workshop cases, we generally weight farther sensors about $10 \times$ stronger than the nearest ones (i.e. even more than the $1 / r$ correction), to reflect the view that the farthest off-
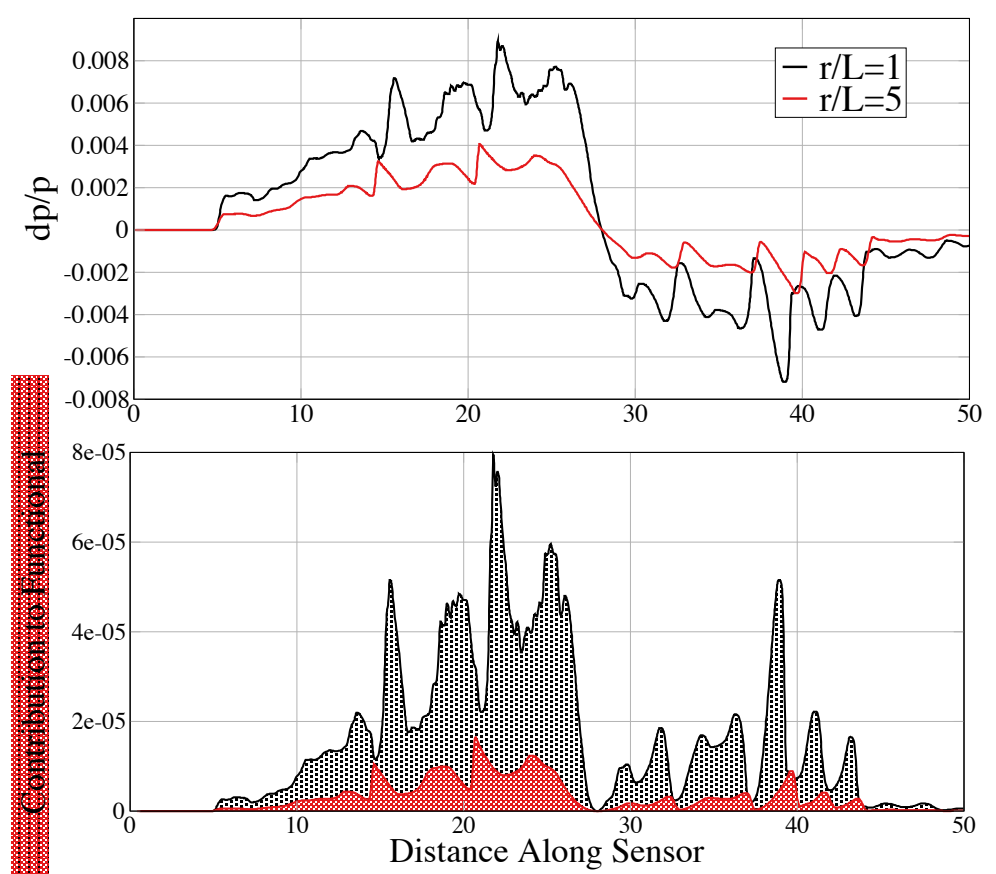

Figure 5: Top: Fine-mesh signatures at two distances for the AXIE case. Bottom: Local unweighted contributions per unit length to the adaptation functional

body signatures are more appropriate inputs for propagating to the ground. This approach is necessarily subjective. The integrated functionals $\mathcal{J}_{r}$ have little direct engineering significance, and are only weakly correlated with the presence and sharpness of pressure fluctuations, and poorly correlated with ground noise.

Finally, weights can help accelerate the mesh adaptation process. By encoding information based on prior experience with a particular problem, the user may avoid a sometimes lengthy discovery process whereby certain physical features are not discovered until much finer meshes. We often apply this type of weighting to thin geometry regions (e.g. the nose) to more quickly resolve the vehicle shape. 
For verification purposes, the workshop also requests the lift, drag, and pitching moment coefficients as computed on the same meshes used for off-body signatures. Since meshes adapted to compute off-body functionals are radically different from meshes adapted to compute aerodynamic loads, we include a term in the adaptation functional to resolve the loads better:

$$
\mathcal{J}_{P}=w_{P}\left(10 C_{D}+C_{L}\right)
$$

where the factor of 10 keeps lift and drag at the same order of magnitude. As these loads are not the main purpose of the workshop, we set $w_{P}$ such that $\mathcal{J}_{P}$ comprises no more than a few percent of the total value of $\mathcal{J}$. For each case we verified our reported loads by computing them much more accurately, using unrotated, unit-aspect ratio meshes adapted only to $\mathcal{J}_{P}$. The loads computed on our finest "boom" meshes are in all cases within $4 \%$ (and usually within $2 \%$ ) of the reference values computed on fine, specialized meshes.

\section{II.B. Local Error Analysis}

To provide an independent assessment of the degree of mesh convergence of the obtained signatures, a procedure based on Richardson extrapolation is used to obtain a local discretization error estimate along each signature. While the adjoint error estimate driving the mesh refinement reflects the total discretization error in the signature functional(s), it does not provide direct insight into the distribution of this error along the signature. The error estimation procedure described below follows the work of Roache, ${ }^{18}$ except we assume that the observed order of accuracy is constant along the signature and can be based on integral quantities. This improves the method's behavior on signatures with sharp variations.

To deduce the distribution of discretization error, consider three signatures from a coarse-medium-fine mesh (c, m, f) sequence, with control volume counts $N_{\mathrm{c}}<N_{\mathrm{m}}<N_{\mathrm{f}}$. The error estimates are based on extrapolation of pointwise differences in a quantity of interest, $f=\Delta p / p_{\infty}$, between the signatures computed on these three meshes, $f_{\mathrm{c}}, f_{\mathrm{m}}, f_{\mathrm{f}}$. Denote the pointwise differences for the coarse-medium signature pair as

$$
\Delta f_{i}^{\mathrm{cm}}=\left(f_{\mathrm{c}}^{\mathrm{m}}-f_{\mathrm{c}}\right)_{i}
$$

for each point $i$ on the coarse-mesh signature $f_{\mathrm{c}}$. The values $f_{\mathrm{c}}^{\mathrm{m}}$ are interpolated from the medium-mesh signature to the coarse-mesh abscissae. Given the non-smooth signatures characteristic of boom problems, we chose to use linear interpolation. For the cases considered in this work, the interpolation error introduced is negligible compared to the actual differences among the signatures.

Error estimates are computed by scaling the local differences using an observed rate of convergence for the sequence of signatures. Following Roache, ${ }^{18}$ we define refinement factors for the coarse-medium and medium-fine mesh pairs:

$$
R_{\mathrm{cm}}=\left(\frac{N_{\mathrm{m}}}{N_{\mathrm{c}}}\right)^{1 / D} \quad \text { and } \quad R_{\mathrm{mf}}=\left(\frac{N_{\mathrm{f}}}{N_{\mathrm{m}}}\right)^{1 / D}
$$

where $D$ is the problem dimension ( $D=3$ here). The observed spatial order of accuracy, $\mathrm{p}$, is then computed by solving

$$
\frac{e_{\mathrm{cm}}}{e_{\mathrm{mf}}}=R_{\mathrm{mf}}^{\mathrm{p}} \frac{1-R_{\mathrm{cm}}^{\mathrm{p}}}{1-R_{\mathrm{mf}}^{\mathrm{p}}}
$$

where $e_{\mathrm{cm}}$ and $e_{\mathrm{mf}}$ are norms of the local solution differences between consecutive signatures. For this work, we use a simple integration of the magnitude of solution differences, using midpoint quadrature:

$$
e_{\mathrm{cm}}=\frac{1}{2} \sum_{i=0}^{S_{c}-1}\left(\left|\Delta f^{\mathrm{cm}}\right|_{i}+\left|\Delta f^{\mathrm{cm}}\right|_{i+1}\right) \Delta \ell_{i}
$$

where $S_{c}$ is the number of points in the coarse-mesh signature. Similarly, $e_{\mathrm{mf}}$ is estimated by using Equations (6) and (9) with the appropriate substitutions for the medium-fine signature pair.

Finally, this observed order of accuracy is applied pointwise to obtain a local discretization error estimate, $E$, for the fine-mesh signature

$$
E_{i}^{\mathrm{mf}}=\frac{1}{R_{\mathrm{mf}}^{\mathrm{p}}-1} \Delta f_{i}^{\mathrm{mf}}
$$

We also compute a coarse-medium local error estimate $E^{\mathrm{cm}}$ based on $\Delta f^{\mathrm{cm}}$ and $R_{\mathrm{cm}}$. 
A comparison of $E^{\mathrm{cm}}$ and $E^{\mathrm{mf}}$ for our AXIE $r / L=5$ signatures is shown in Figure 6. Both error estimates indicate lower discretization error over the front half of the signature and higher error over the back half. The right frame of Figure 6 shows that $E^{\mathrm{cm}}$ and $E^{\mathrm{mf}}$ are generally consistent in magnitude and sign, which is an indication that the mesh sequence may be near the asymptotic regime. Moreover, $E^{\mathrm{cm}}$ generally bounds the fine mesh solution. For this case, even the coarser error estimate is reliable.

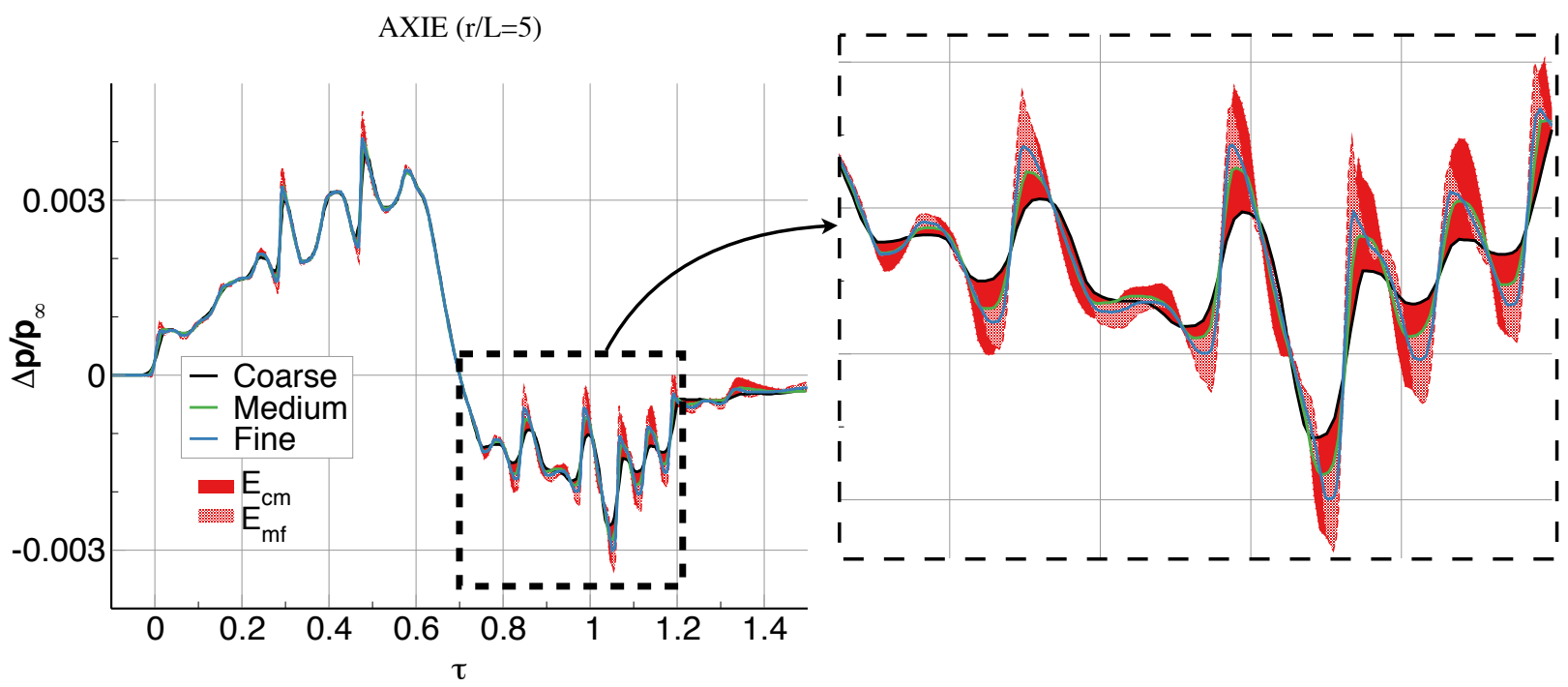

Figure 6: AxIE solutions at $r / L=5$, with detailed breakdown of discretization error estimates. Left: Coarse, medium and fine mesh solutions, and local discretization error estimates from Equation (10) (coarse-medium $E_{c m}$ and medium-fine $\left.E_{m f}\right)$. Right: Aft-region zoom, showing that $E_{c m}$ generally bounds the fine mesh solution.

Depending on the consistency of these two consecutive error estimates, we assign it a degree of confidence. Where $E_{i}^{\mathrm{cm}}$ and $E_{i}^{\mathrm{mf}}$ have the same sign, we plot $E_{i}^{\mathrm{mf}}$ as the local error estimate, and label it "High Confidence". Where the signs are different, we plot both $E^{\mathrm{mf}}$ and $E^{\mathrm{cm}}$ to indicate the uncertainty in direction, and label it "Medium Confidence". Figure 7 shows these error estimates for our well-behaved AXIE $r / L=5$ signatures.

This error estimation approach assumes that the differences in the solutions are getting smaller $\left(e_{\mathrm{mf}} \leq e_{\mathrm{cm}}\right)$, which must be true to apply asymptotic analysis (specifically Equation (10)). If instead $e_{\mathrm{mf}} \geq e_{\mathrm{cm}}$, then we assume first-order convergence (set $p=1$ in Equation (10)). Depending on whether the final two signatures are near the asymptotic range, this may or may not be valid, and is therefore la-

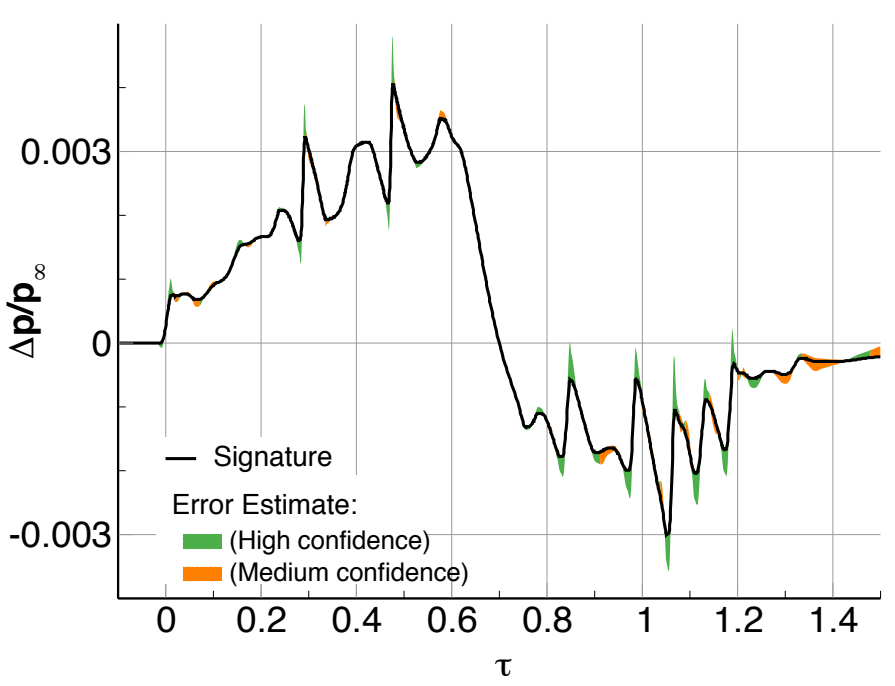

Figure 7: High- and medium-confidence discretization error estimates plotted with the fine mesh signature for AXIE at $r / L=5$ beled "Low Confidence".

To elucidate the accuracy and limitations of this approach, consider a more challenging test case: the $\mathrm{C} 25 \mathrm{~F}$ at $r / L=5$ and $\Phi=0^{\circ}$, as computed on a three-mesh sequence ending in $18 \mathrm{M}$ cells. Figure 8 compares the error estimates on the fine mesh to a reference solution from a mesh with over 20 times as many cells. There is broad qualititative agreement; in most locations, the error estimate provides a good indication of the characteristics of the reference solution. In the front region of the signature, the errors estimates are 


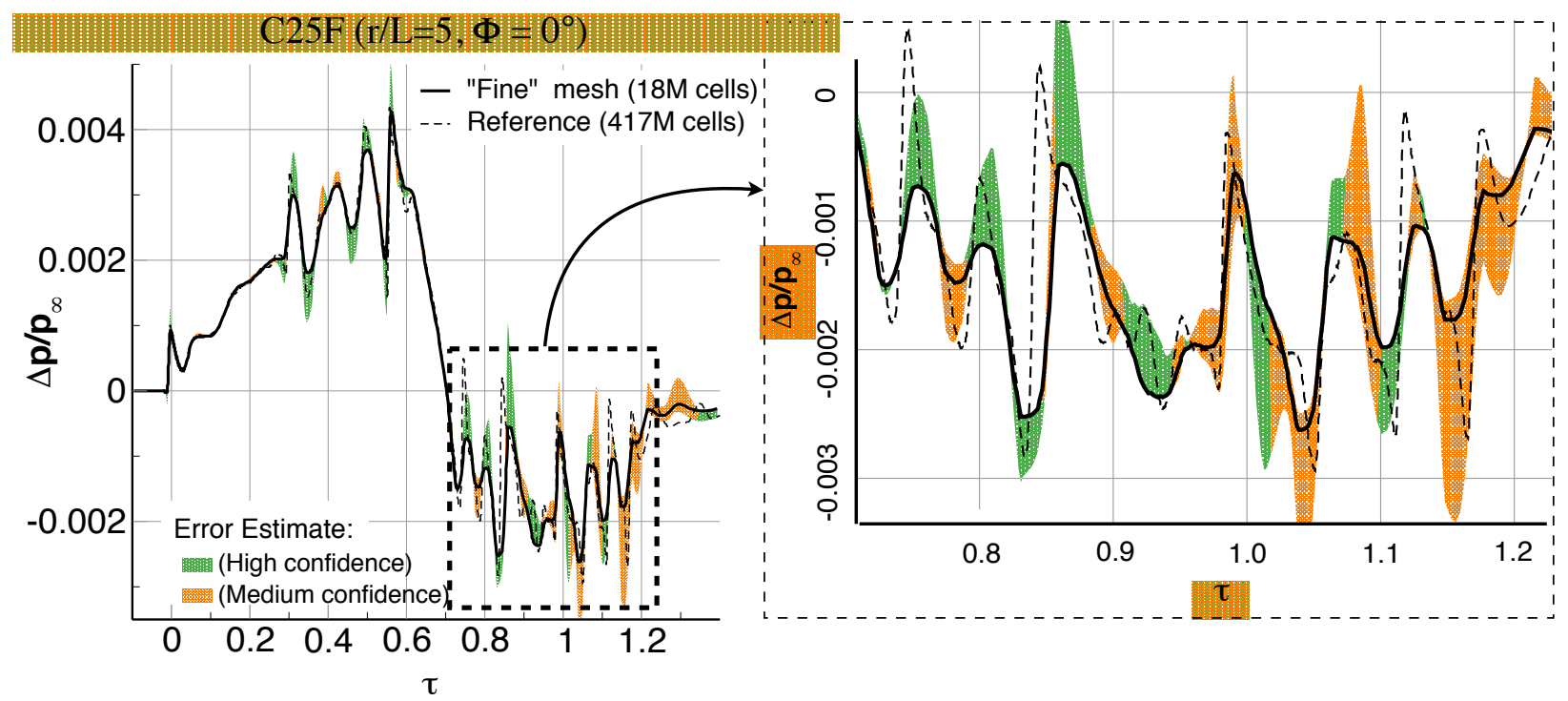

Figure 8: Left: Discretization error estimates on $\mathrm{C} 25 \mathrm{~F}$ at $r / L=5$ and $\Phi=0^{\circ}$ compared to a signature computed on an extremely fine reference mesh with $23 \times$ more cells. Right: Aft-region zoom, showing that, despite the relative coarseness of the mesh, the error estimates correctly highlight the uncertain regions, generally get the direction correct, and have roughly the correct magnitude.

smaller, indicating deeper spatial convergence, and they bound the reference mesh solution. In the aft region, the error estimates indicate less convergence. In this region, the error estimates do not reliably predict the error bounds on the extrema of the signature and exhibit sensitivity to phase shifts (e.g. at $\tau=0.85$ ).

The main purpose of this analysis is to identify regions of the signature with the greatest sensitivity to mesh refinement and to flag mesh sequences with poor spatial convergence characteristics. Experience with a large number of datasets for numerous solvers and meshing approaches indicates that this approach consistently provides a useful qualitative assessment of the behavior of the signature with mesh refinement. Although it can be used with any mesh refinement approach, this technique is especially useful when employed alongside adjoint-based mesh refinement, as we do in this work. By reducing discretization error in the global adaptation functional $\mathcal{J}$, the adjoint is indirectly driving out local errors in the signature, albeit at different rates. Local information on convergence is therefore implicitly available in the solution sequence. Although we do not do so in this work, this information could be used to adjust the weights in the global functional to better balance the accuracy across the entire signature.

\section{II.C. Atmospheric Propagation and Noise Metrics}

Atmospheric propagation for all submitted cases was performed with NASA's SBOOM code (v2.5). ${ }^{19}$ This propagation tool was specifically developed for boom analysis. It uses acoustical ray tracing to compute the sound trajectory through the atmosphere, and then uses an augmented Burgers' equation solver to compute the evolution of the pressure signal as it descends through the atmosphere. The approach captures not only non-linear wave propagation, but also thermo-viscous absorption and molecular relaxation of nitrogen and oxygen, the primary constituents of the atmosphere. The governing equations are solved as a quasione-dimensional PDE along the ray tube in the time-domain. The system is solved numerically using a second-order finite-difference discretization with operator splitting. ${ }^{19}$

Noise metrics for all ground signatures were computed using NASA's "Loudness Code for Asymmetric Sonic Booms" (LCASB), which is used broadly throughout both government and industry. ${ }^{20}$ LCASB takes the time-domain ground signature as input and computes standard noise metrics that are widely used for boom assessment: the A- and C-weighted Sound Exposure Level (ASEL, CSEL) and the Perceived Level of noise (PL), according to the Stevens Mark VII frequency-weighting. ${ }^{21}$ 


\section{Nearfield Results}

We solve all four nearfield cases from SBPw2. The two primary geometry models are based on a full low-boom configuration by NASA called "Concept 25D" (see Figure 2). This configuration is derived from a vehicle designed for low on-track ground loudness using the Cart3D design framework. ${ }^{12}$ Two versions of this model are used in the workshop. The first has a flow-through nacelle $(\mathrm{C} 25 \mathrm{~F})$, and the second includes a more realistic propulsion system with a plug nozzle (C25P). The two are largely similar, but have different fuselage profiles and different vertical tail bulb contours. The two lower-complexity models (AXIE and JWB were independently designed to produce on-track signatures at $r / L=3$ that are broadly similar to that of the $\mathrm{C} 25 \mathrm{~F}$. Table 1 summarizes the flight conditions and reports the aerodynamic loads computed on our finest meshes.

Table 1: Flight conditions and computed performance metrics for nearfield cases

\begin{tabular}{|c|c|c|c|c|c|c|c|c|c|}
\hline & \multirow[b]{2}{*}{ Case } & \multicolumn{3}{|c|}{ Flight Conditions } & \multicolumn{5}{|c|}{ Inviscid Aerodynamic Coefficients* } \\
\hline & & Mach & Altitude (km) & $\alpha\left(^{\circ}\right)$ & $C_{L}$ & $C_{D}$ & $C_{M}^{\dagger}$ & $L(\mathrm{~m})$ & $A_{\text {ref }}\left(\mathrm{m}^{2}\right)$ \\
\hline I. & AXIE & 1.6 & 15.76 & 0 & - & - & - & 32.92 & 37.16 \\
\hline II. & JWB & 1.6 & 15.76 & 2.3067 & 0.077 & 0.0072 & -0.066 & 38.70 & 65.60 \\
\hline III. & $\mathrm{C} 25 \mathrm{~F}$ & 1.6 & 15.76 & 3.375 & 0.068 & 0.0091 & -0.052 & 32.92 & 37.16 \\
\hline IV. & $\mathrm{C} 25 \mathrm{P}$ & 1.6 & 15.76 & 3.375 & $0.058^{\ddagger}$ & $0.0093^{\ddagger}$ & $-0.043^{\ddagger}$ & 32.92 & 37.16 \\
\hline
\end{tabular}

For AXIE, on-track signatures are requested at $r / L=\{1,3,5\}$. The three other cases request discretized boom carpets, with signatures at $\Phi=\left\{0^{\circ}, 10^{\circ}, 20^{\circ}, 30^{\circ}, 40^{\circ}, 50^{\circ}\right\}$ at multiple radial locations: $r / L=\{0.85,2.55\}$ for JWB and $r / L=\{1,3,5\}$ for the other three cases, ${ }^{\mathrm{c}}$ with $L$ being the reference lengths given in Table 1. For each of the sensor locations, a sequence of refined solutions is to be provided (coarse-medium-fine). In all, for complete participation, 51 sensor locations are submitted, each consisting of signatures at three resolutions.

\section{III.A. Surface Geometry}

For surface geometry, continuous surface definitions were provided in the form of STEP (*.stp) files. ${ }^{22}$ However, we opted to use the boundary patches of the finest unstructured inviscid workshop grids. These tessellations are noticeably irregular. Figure 9 shows how spurious pressure disturbances are generated at irregularities in the surface mesh for Case 1 (AXIE). These appear to be most severe at vertices that do not touch six edges, as shown in the top right frame of Figure 9. These fluctuations are irregularly spaced and can persist far from the body, especially with a low-dissipation flow solver. Indeed they are still present at $r / L=1$, as evident in the plot in Figure 9.

The surface resolution seems adequate, although it depends on the resolution of the unstructured meshes (see Table 2). To verify that this was not the main problem, we tried refining the AXIE surface grid by Loop subdivison with smoothing. This did not remove the problems, indicating that the problem was indeed regularity, not resolution. (The subdivision scheme does not remove the irregularities). We then developed a more regular surface grid by revolving the radius distribution provided by the workshop (Figure 9, bottom left). Because this surface grid is coarser than the flow mesh, small pressure fluctuations still arise at each vertex as the faceted surface representation cuts through the Cartesian mesh. However, these fluctuations are regularly spaced and cancel quickly, which leads to a smoother signature at $r / L=1$ (red curve in Figure 9).

Table 2: Discrete geometry resolution $\left(N_{\triangle}\right.$ is the number of triangles)

\begin{tabular}{rlc}
\hline & Case & $N_{\triangle}$ \\
\hline I. & AXIE & $274 \mathrm{~K}$ \\
II. & JWB & $745 \mathrm{~K}$ \\
III. & C25F & $1.82 \mathrm{M}$ \\
IV. & C25P & $1.20 \mathrm{M}$ \\
\hline
\end{tabular}

${ }^{\mathrm{c}}$ The JWB normalized distances are different, but because of the reference length in Table 1 , the absolute distances $r$ are the same as $r / L=\{1,3\}$ for the other cases. 


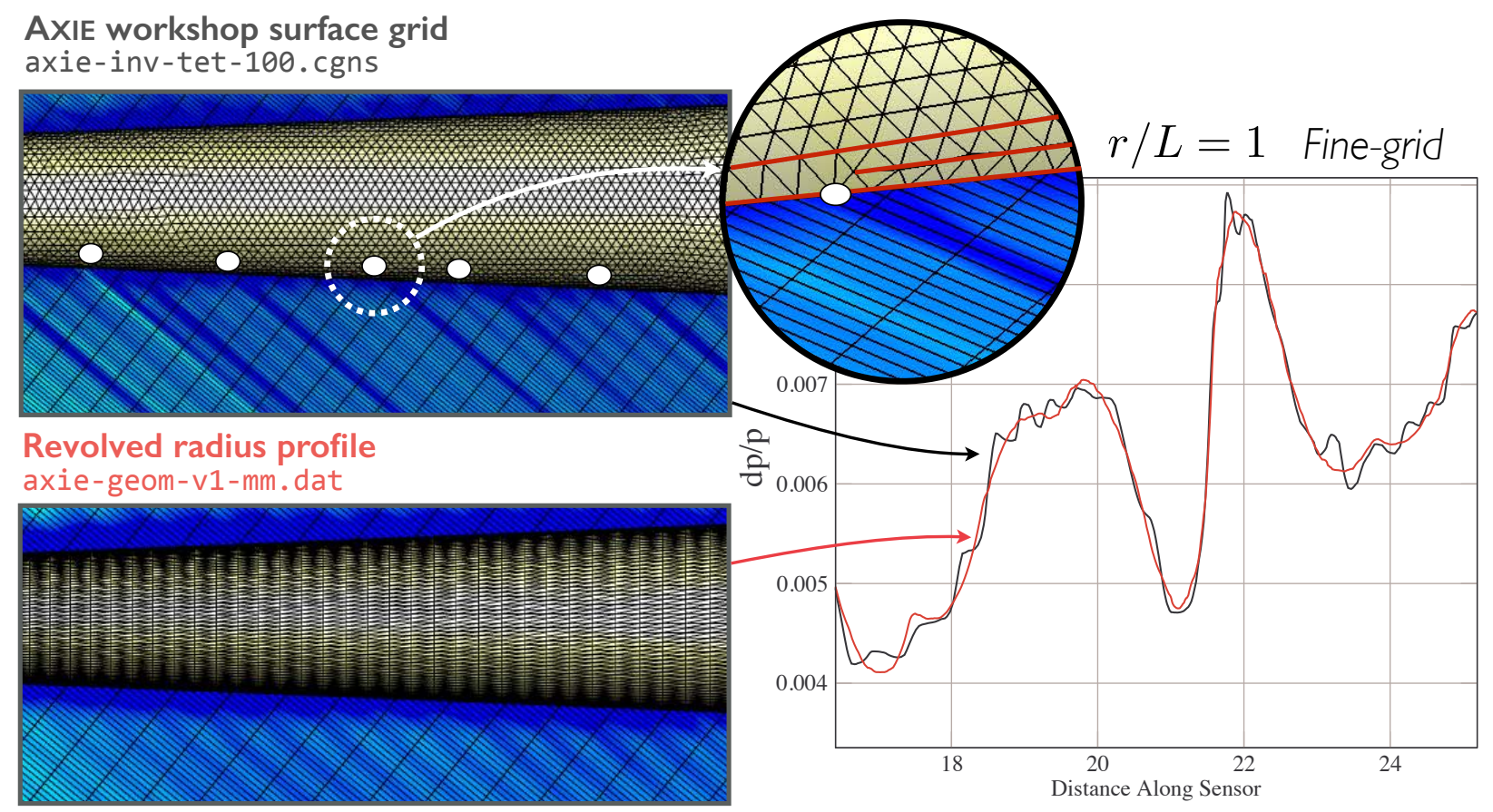

Figure 9: Top: Irregular vertices in the provided unstructured surface grids cause local asymmetry and generate spurious pressure disturbances that persist far from the body right, black line. Bottom: A more regular tessellation still generates fluctuations, but they quickly cancel, leading to a smoother $r / L=1$ signature (right, red line).

The tessellation artifacts become insignificant by $r / L=3$. Since $r / L=1$ is not an especially relevant distance for propagation, we opted to use the workshop surface grids, rather than develop custom surface grids from the continuous definition. This also has the advantage of keeping our surface representation consistent with participants using the workshop grids.

\section{III.B. Case I: AXIE - Axisymmetric Body}

The axisymmetric body of revolution was designed to have an off-body pressure signature similar to the C25F on-track signature. The relevant (i.e. designed) portion of the geometry extends from the nose to about $x=40 \mathrm{~m}(\tau=1.2)$, after which it maintains a constant radius for about another $50 \mathrm{~m}$ and then smoothly closes out.

\section{Meshing}

The left frame of Figure 10 shows the initial mesh with about $22 \mathrm{~K}$ control volumes. This initial mesh is given higher resolution along the front portion of each signal and near the body to ensure that the adaptation starts with a sufficiently good representation of the geometry and initial flow features. Thereafter, the mesh is adapted to a weighted sum of functionals (Equation (3)) for each of the three sensors shown as blue lines in Figure 10. The functional weights in Equation (4) are $w_{1}=0.5, w_{3}=3, w_{5}=10$ - this both corrects for the natural decay in pressure with distance and also emphasizes the farther sensors. For this case, we did not apply any special weighting profile $w(\ell)$ along the sensor, other than linearly tapering the weight to zero from $\tau=1.76$ to $\tau=1.82$. We also specified a very short, low-weight sensor immediately under the thin nose to accelerate the adaptation there. The middle frame of Figure 10 shows an intermediate mesh, where the adaptation has added resolution in the most significant regions of the signature. 


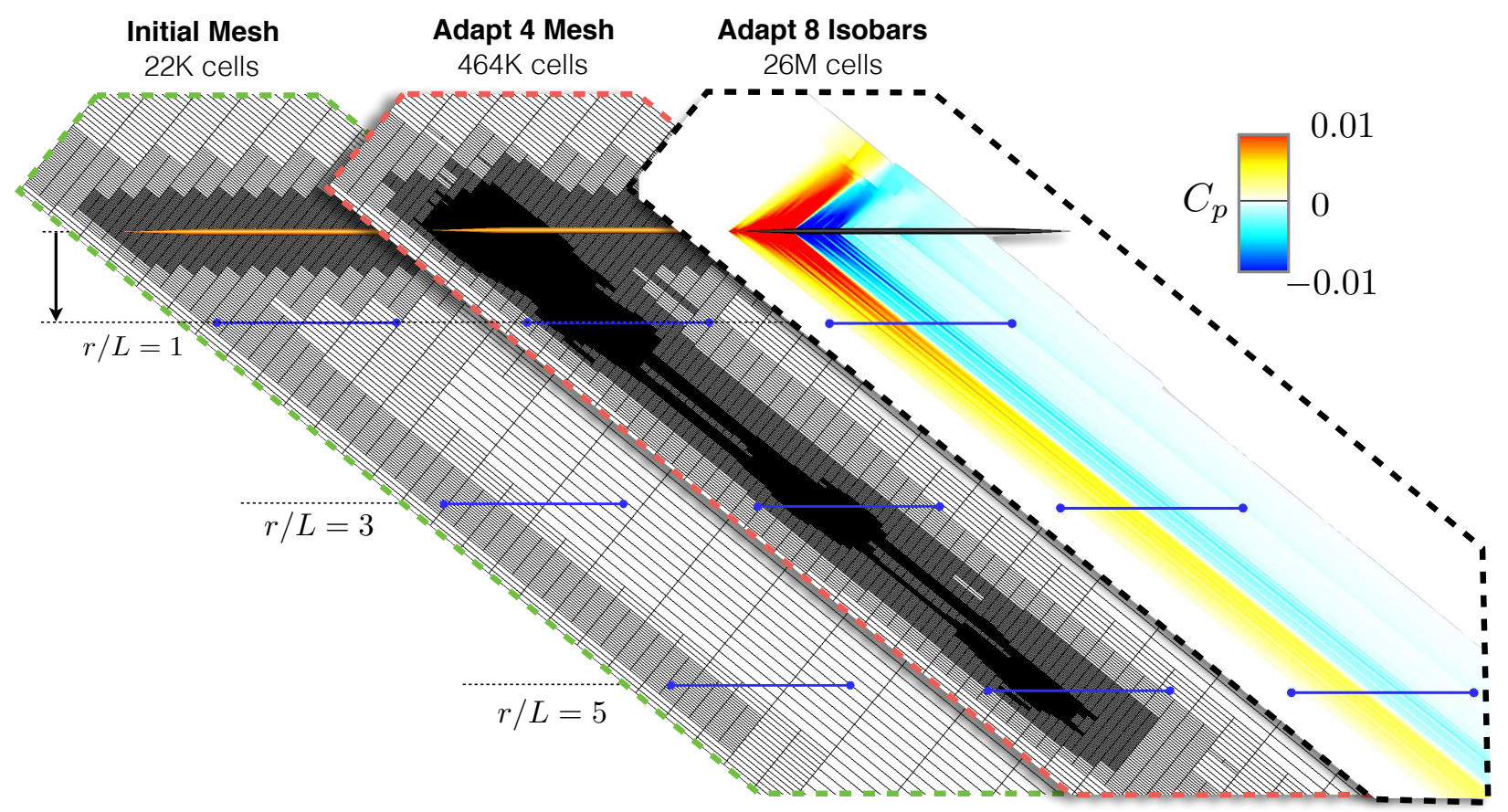

Figure 10: AXIE symmetry plane meshes (initial grid and fourth adapted mesh) and isobars on the finest mesh.

\section{Results}

The right frame of Figure 10 shows isobars for the final flow solution on the fine mesh. From this distance only the general high-pressure to low-pressure trend is visible. Figure 11 shows the three pressure signatures, scaled by $\sqrt{r / L}$ to remove the natural pressure decay and reveal the evolution of signal features with distance from the body. The signal exhibits the expected behavior - high/low pressure regions appear to "move" forward/back, due to the slightly different local Mach numbers. Smoother compressions steepen and form shocks, while the main expansion flattens out. In this scaling, the signatures at all distances look quite similar, although $r / L=1$ exhibits the spurious high-frequency oscillations explained earlier.

\section{Mesh Convergence}

As shown previously in Figure 7, the coarse, medium and fine signatures at $r / L=5$ are well-behaved and asymptotically converging. We then took these signals and propagated them to the ground using SBOOM. Figure 12 shows that the propagated ground signals are visually indistinguishable. Indeed, the computed noise metrics, shown in Table 3 , vary only by about $0.4 \mathrm{~dB}$ in PL between the coarse and fine CFD meshes. All together, these results give us reasonably high confidence in the submitted signatures. As shown in Table 5, the final mesh size of $26 \mathrm{M}$ control volumes is about half the size of the finest workshop mesh. The case required approximately 75 minutes on a single compute node with 28 cores $^{\mathrm{d}}$ and 128 GB of main memory.
AXIE (fine mesh)

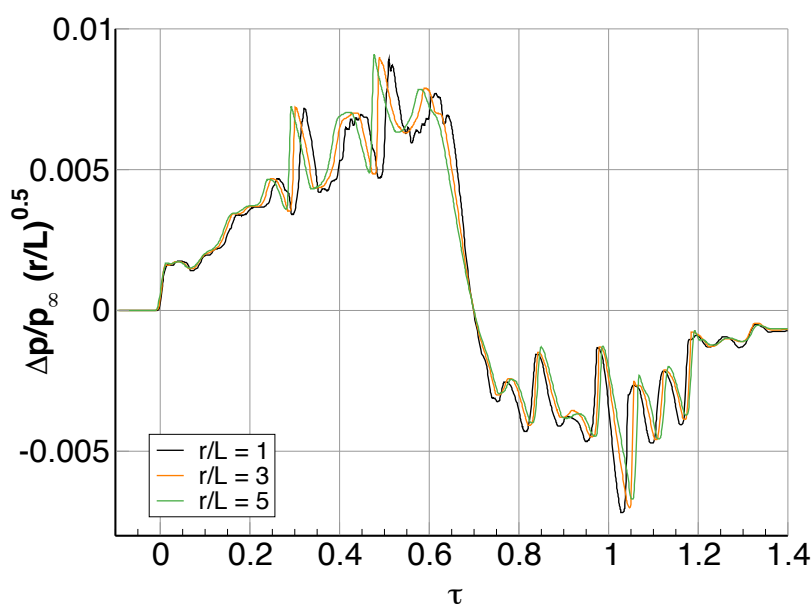

Figure 11: AXIE pressure signature "aging" with distance from vehicle fine mesh, $26 \mathrm{M}$ cells

\footnotetext{
${ }^{\mathrm{d}}$ Intel Xeon E5-2680v4
} 


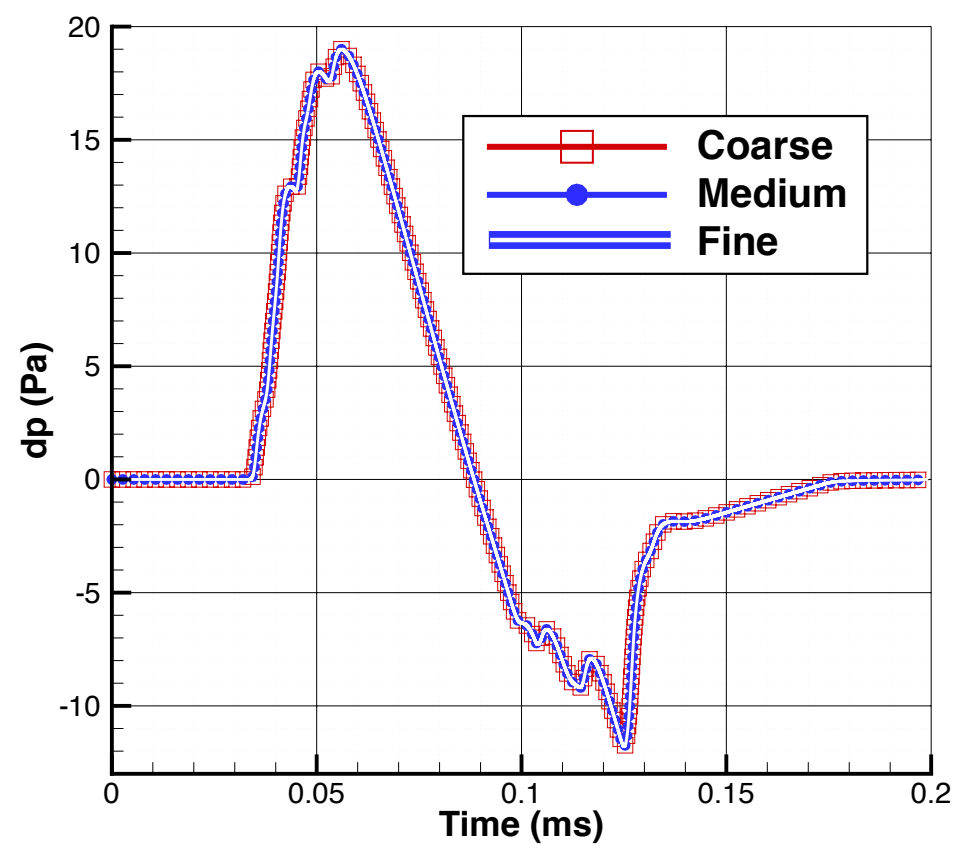

Figure 12: AXIE ground signatures propagated from $r / L=5$ on coarse, medium and fine meshes.

\section{III.C. Case II: JAXA Wing-Body}

The JWB is a wing-body geometry developed by JAXA. It was designed to approximately match an on-track equivalent area distribution derived from the C25 models. ${ }^{23}$ Following the approach described in Section II.A, we solve each of the six azimuthal angles on its own mesh, which is aligned with both the off-track angle and the Mach angle (Equation (2)). The functional weights in Equations (4) and (5) are $w_{0.85}=0.1$ and $w_{2.55}=1$, with $w_{P}$ set so that $\mathcal{J}_{P}$ comprises about $1 \%$ of the total functional value.

Early meshing studies revealed that the aft region of the on-track signature, around $\tau=0.97-1.0$, is strongly sensitive to the mesh (see Figure 15, top left frame). A sequence of large oscillations in that region initially would consistently emerge on quite fine meshes (at least $100 \mathrm{M}$ cells). A qualitative investigation indicated that the oscillations originated in the wake. To investigate more precisely where these features originate, we specified an adaptation functional for only the $\tau=0.97-1.0$ segment of the sensor, and solved the adjoint equations against this functional. Figure 13 shows the magnitude of this adjoint solution plotted on the lower surface of the vehicle. The highlighted regions of the surface are those that play the most signifant role in this segment of the signature. The figure suggests that wing tip effects play an important role in the formation of these aft oscillations. Indeed, Mach cones opening backwards from the wingtips intersect the wake at precisely the location where the oscillations originate.

These features proved to be challenging to resolve on coarse meshes. We increased the initial mesh resolution near the wing tips and and boosted the local weight $w(\ell)$ in the adaptation functional (see Figure 14). In the end, we selected the lowestresolution mesh sequence that began to capture the oscillations (though not in their full magnitude). The submitted coarse, medium and fine meshes had on average 6,9 , and $32 \mathrm{M}$ cells per off-track angle (exact cell counts vary), which is substantially finer than the finest workshop-provided mesh.

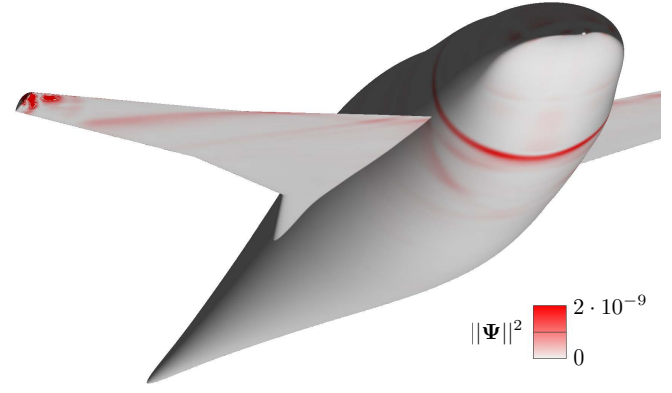

Figure 13: JWB: An adjoint solution highlights the regions on the lower wing and fuselage that most contribute to the region of the signature, $0.97<\tau<1\left(r / L=2.55, \Phi=0^{\circ}\right)$. Showing local sum of squares of adjoint variables, as computed on a very fine mesh (228 M cells).

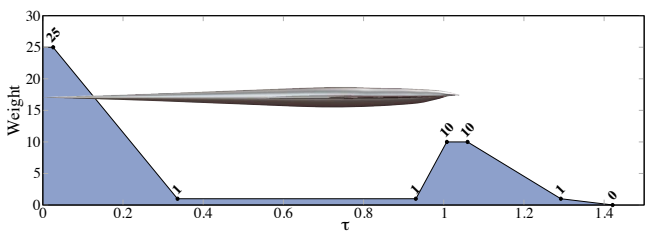

Figure 14: JWB: Weight profile $w(\ell)$ in Equation (3) used to help nose and wake regions be resolved earlier in adaptation 

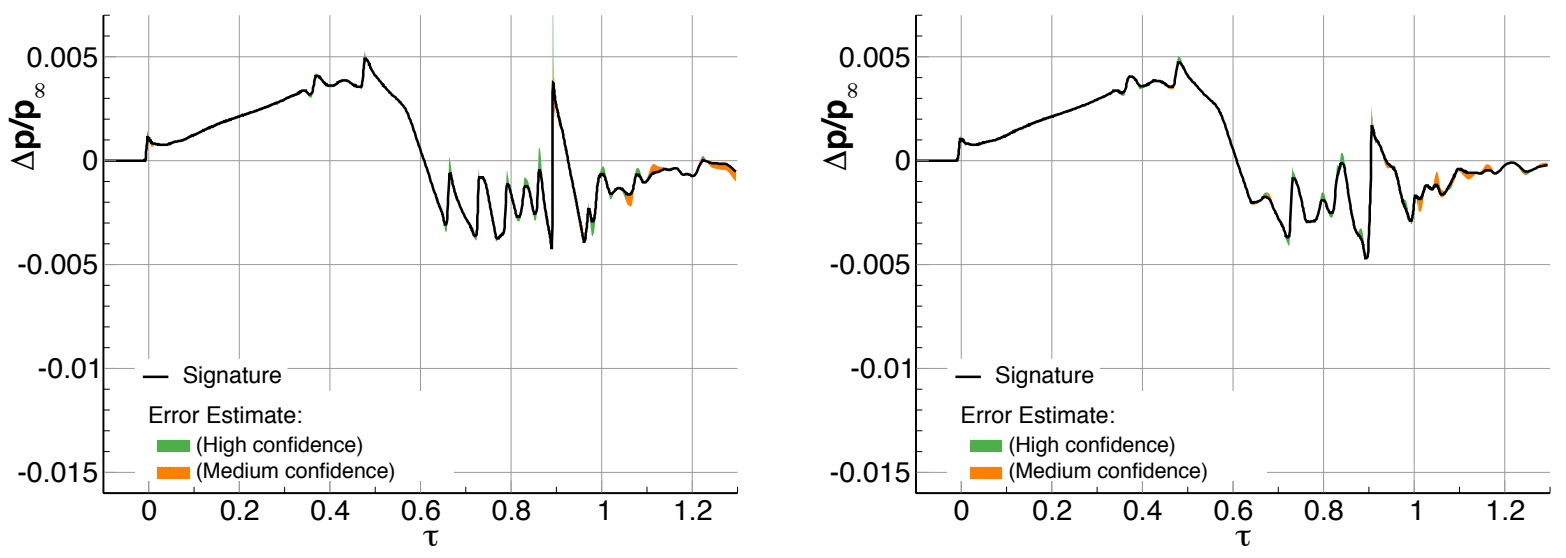

$\operatorname{JWB}\left(\mathrm{r} / \mathrm{L}=2.55, \Phi=20^{\circ}\right)$

$\operatorname{JWB}\left(\mathrm{r} / \mathrm{L}=2.55, \Phi=30^{\circ}\right)$
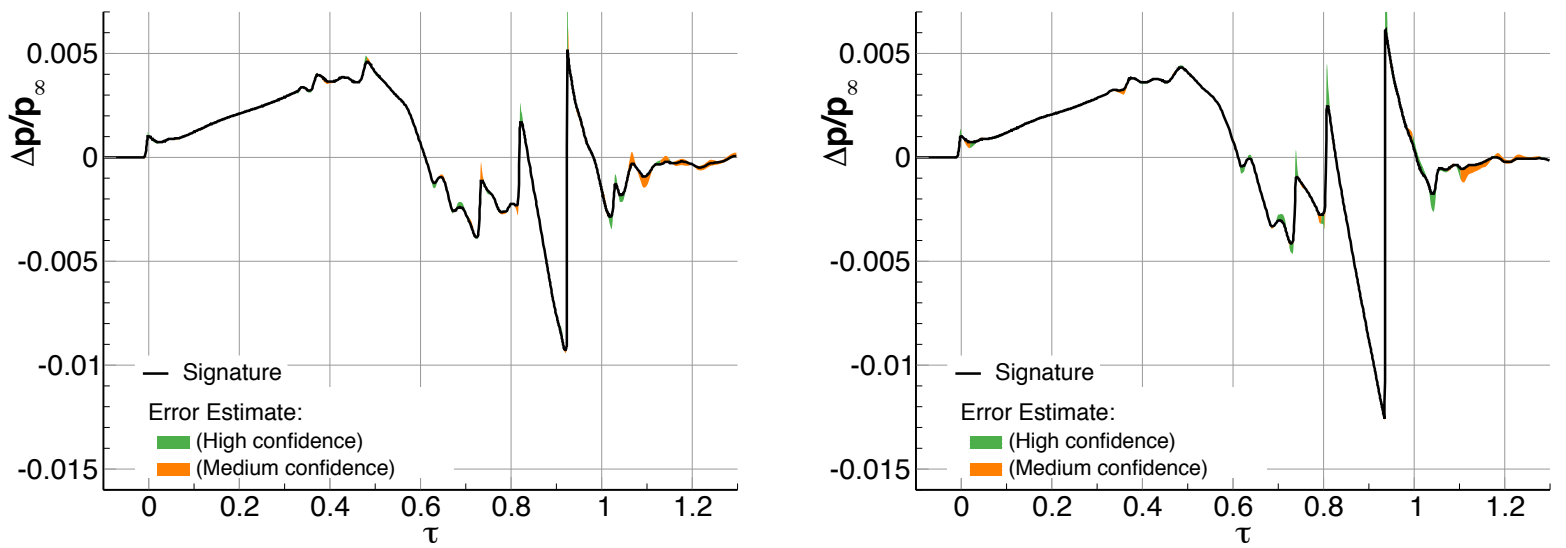

$\operatorname{JWB}\left(\mathrm{r} / \mathrm{L}=2.55, \Phi=40^{\circ}\right)$

$\operatorname{JWB}\left(\mathrm{r} / \mathrm{L}=2.55, \Phi=50^{\circ}\right)$
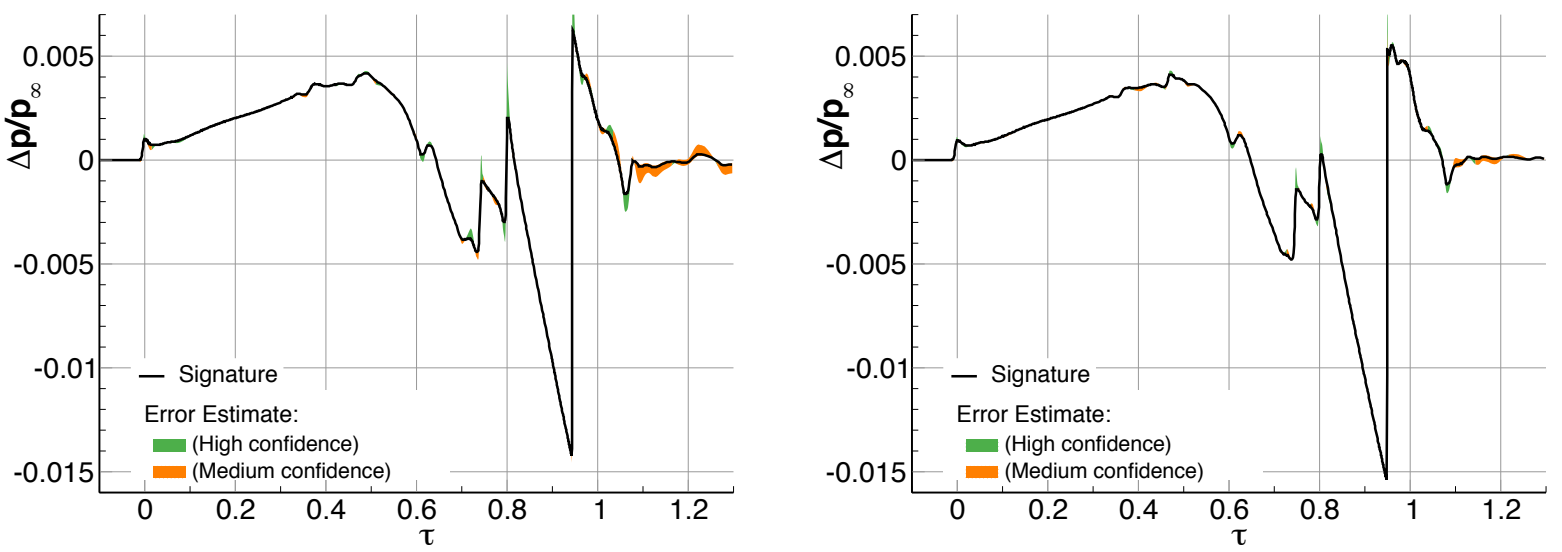

Figure 15: JwB: Pressure signature carpet at $r / L=2.55$, showing fine mesh signature and local error estimates $\left(E^{\mathrm{mf}}, E^{\mathrm{cm}}\right) . x$-axis shows $\tau=(x-r \beta) / L$. To stabilize error estimates at $\Phi=\left\{30^{\circ}, 40^{\circ}\right\}$, coarse meshes were replaced by "very coarse" meshes. 
Figure 15 shows the fine mesh solutions at $r / L=2.55$. Overall, the on-track signature is more compact than the off-track signatures, which have a strong aft shock. This is likely a consequence of a primarily on-track design. ${ }^{23}$ Figure 15 also shows local error estimates for the finest meshes, computed using the technique in Section II.B. The error estimates are generally extremely sharp across most of the signature, except in the wake region. While most of the signature was well resolved even on our coarse meshes, the aft oscillations (see the $\Phi=0^{\circ}$ signature) emerged only on the finest mesh. The extrapolation-based error estimates correctly indicate that this region is much farther from convergence than the front.

\section{III.D. Case III: C25F}

The C25D low-boom model is the most complex required case for SBPW2. As shown in Figure 2, it is a nearly complete configuration, including a fuselage, wings, empennage (with a shaped bulb on the vertical tail). To remove the complexity of powered boundary conditions, this model includes a simplified flow-through nacelle.

We follow the same meshing approach as before. Six meshes are generated, one for each azimuth. The adaptation functional and weights are roughly the same as those used for the previous cases $\left(w_{1}=1, w_{3}=3\right.$, $w_{5}=10$, and $w(\ell)$ similar to Figure 14).

As with the previous cases, we initially approached this case with deep mesh adaptation studies to establish reference solutions and identify meshing requirements. Figure 16 shows the convergence of the adaptation functional in one such study, which used up to about $300 \mathrm{M}$ cells. To determine which signatures to submit, we used three main criteria:

1. Convergence of the value of the adaptation functional $\mathcal{J}$, as shown in Figure 16.

2. Generally decreasing adjoint-based error estimates (see error bars in Figure 16)

3. Presence of the qualititative features of a very fine reference mesh, although without yet sharply resolving them (demonstrated earlier in Figure 8)

Based on these criteria, we submitted sequences of meshes with on average 4, 8, and $19 \mathrm{M}$ cells per azimuth, indicated roughly by the boxed region in Figure 16. Figure 17 shows the fine mesh $r / L=5$ signatures at each azimuth. The ontrack signature has a substantially tighter envelope of pressure variations, and quite detailed aft pressure fluctuations. Offtrack, the signatures exhibit larger shocks and a simpler overall structure, reflecting the fact that it was designed primarily for low on-track noise. ${ }^{12 \mathrm{e}}$

Figure 17 also shows localized error estimates based on the approach in Section II.B. This provides a more stringent eval-

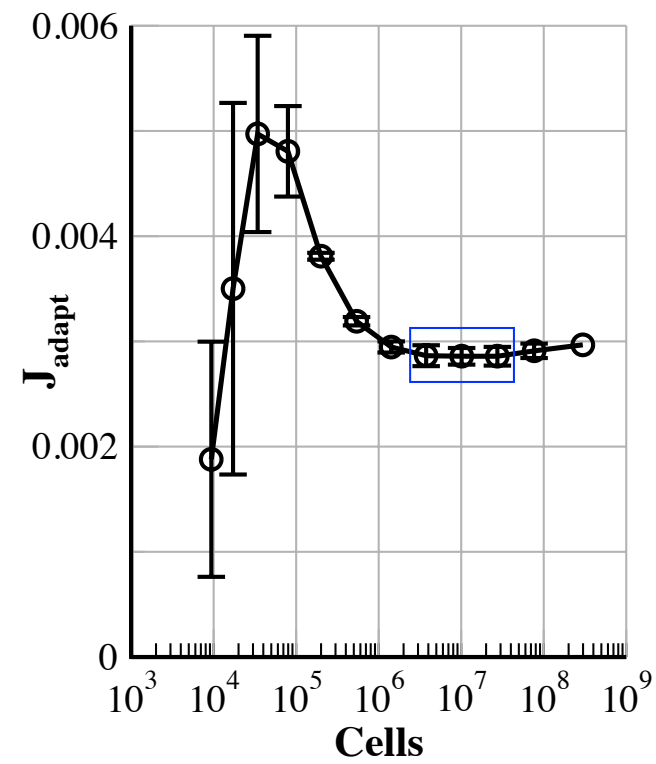

Figure 16: C25F: Convergence of $\mathcal{J}$ with mesh adaptation for an initial refinement study $(\Phi=$ $0^{\circ}$ mesh). Bars indicate an evolving adjointbased estimate of the discretization error in the functional value. Blue box shows the levels chosen for submission as coarse-medium-fine. uation of our choice of mesh resolution. (This error analysis technique was developed after completing the workshop submissions and therefore did not inform our submissions.) The error estimates indicate that the submitted sequence is much less mesh converged than for the JWB case ( $c f$. Figure 15). At some azimuths, the estimates are first-order ("Low Confidence"), because the integrated solution differences, $e$, were not yet decreasing (see Equation (10)).

This more localized view reveals a potential pitfall of monitoring convergence only of a global adaptation functional, $\mathcal{J}$. Convergence of the value of $\mathcal{J}$ can conceal quite significant local variations, which are revealed by the local error estimate $E$. With this enriched information, one might conclude in retrospect that additional mesh refinement may have been appropriate, depending on the degree to which the errors impact the noise metrics.

\footnotetext{
e Note also that due to modeling issues in format conversion, the workshop geometry is a somewhat different (louder) design than published in that paper.
} 
$\mathrm{C} 25 \mathrm{~F}\left(\mathrm{r} / \mathrm{L}=5, \Phi=0^{\circ}\right)$

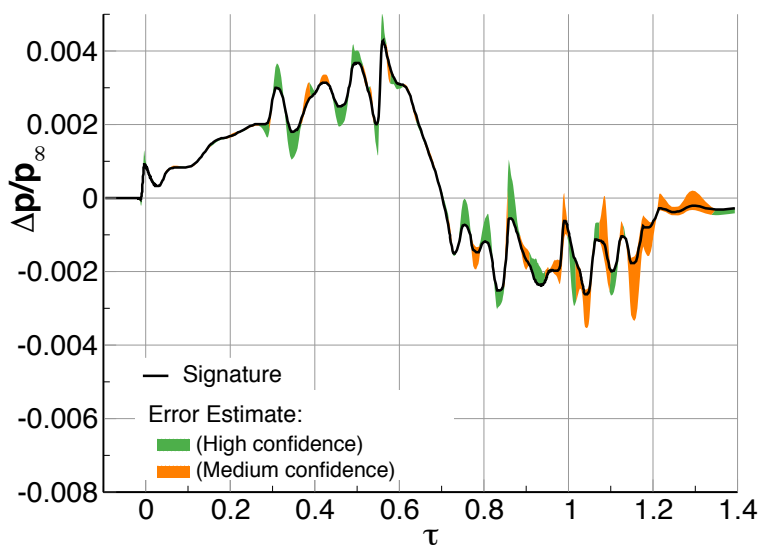

$\mathrm{C} 25 \mathrm{~F}\left(\mathrm{r} / \mathrm{L}=5, \Phi=20^{\circ}\right)$

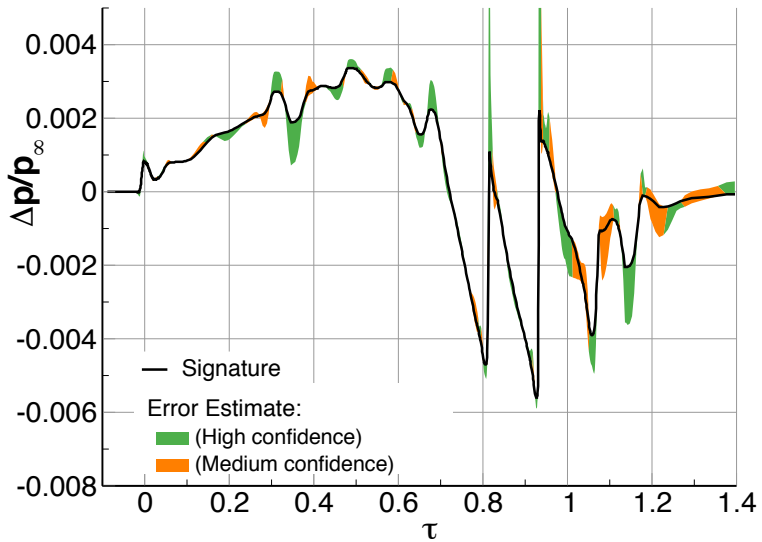

$\mathrm{C} 25 \mathrm{~F}\left(\mathrm{r} / \mathrm{L}=5, \Phi=40^{\circ}\right)$

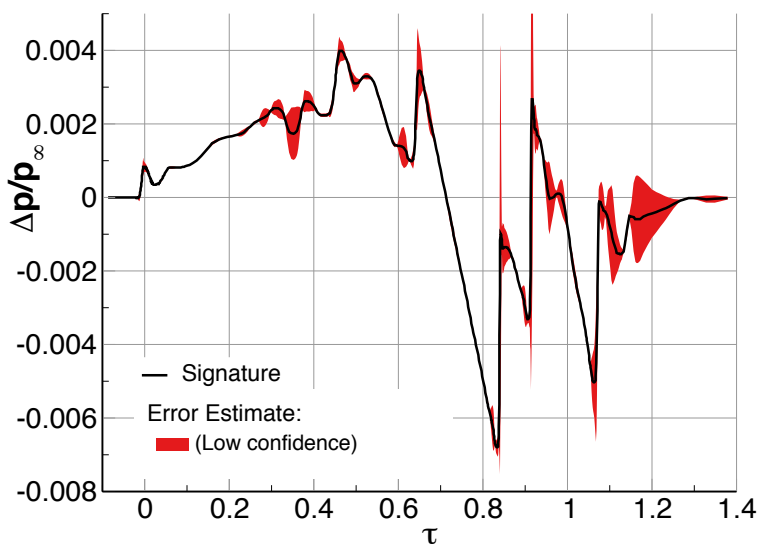

$\mathrm{C} 25 \mathrm{~F}\left(\mathrm{r} / \mathrm{L}=5, \Phi=10^{\circ}\right)$

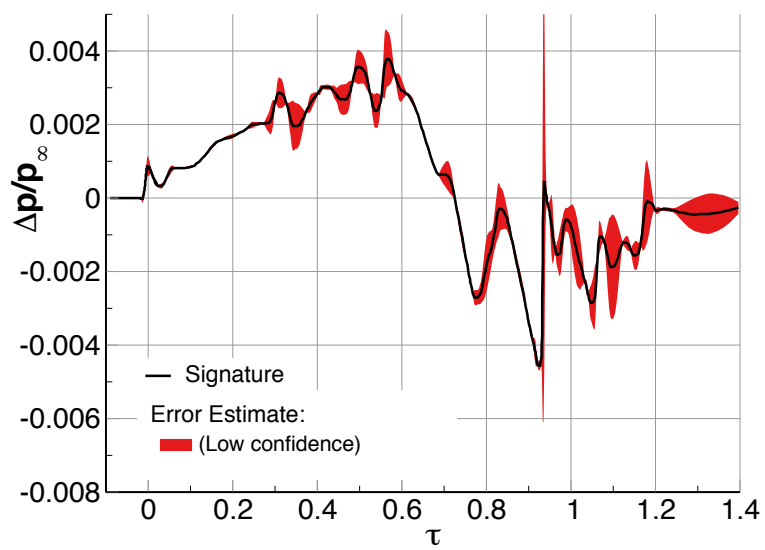

$\mathrm{C} 25 \mathrm{~F}\left(\mathrm{r} / \mathrm{L}=5, \Phi=30^{\circ}\right)$

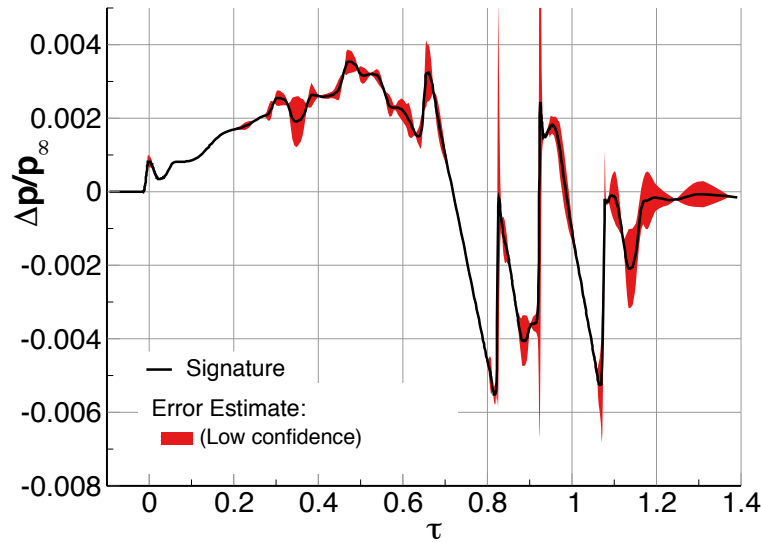

$\mathrm{C} 25 \mathrm{~F}\left(\mathrm{r} / \mathrm{L}=5, \Phi=50^{\circ}\right)$

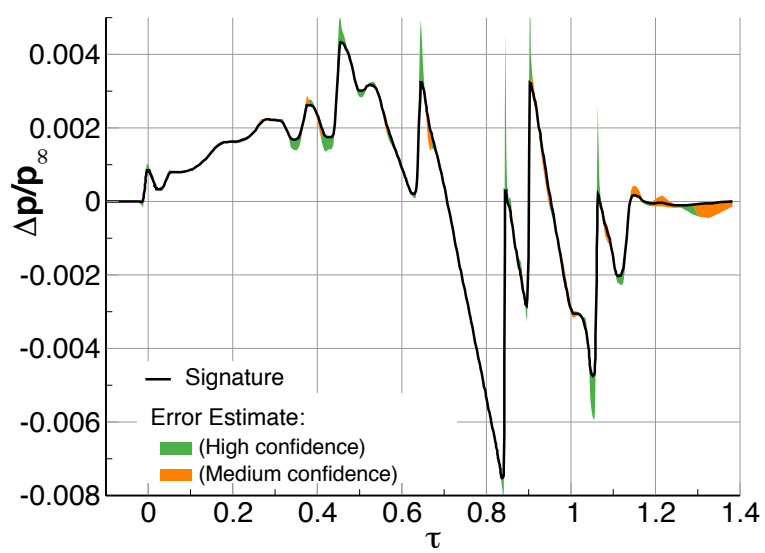

Figure 17: C25F: Pressure signature carpet at $r / L=5$, showing fine mesh signature and local error estimates $\left(E^{\mathrm{mf}}, E^{\mathrm{cm}}\right) . x$-axis shows $\tau=(x-r \beta) / L$. 


\section{III.E. Case IV: C25P}

The $\mathrm{C} 25 \mathrm{P}$ is a variant of the $\mathrm{C} 25 \mathrm{~F}$ with powered boundary conditions modeling the propulsion system. The geometry is substantially similar to the flow-through version, but the fuselage and tail bulb have both undergone re-contouring as a result of design optimization for powered flight. ${ }^{12}$ The inlet back-pressure ratio is $p / p_{\infty}=3.2606$, while the exhaust conditions are based on stagnation properties (at fixed $\gamma$ ): $p_{t} / p_{\infty}=14.540$ and $T_{t} / T_{\infty}=7.8722$.

This case involves more complex flow physics in the plume, as illustrated in the density contours in Figure 18. Pressure disturbances from the empennage pass through the plume, suggesting that resolution of at least the initial portion of the plume is important for computing off-body signatures. The plume effectively lengthens the geometry and expands the zone of influence, which increases the meshing requirements. An additional challenge for mesh refinement is the fact that the plume shape evolves as the mesh is adapted.

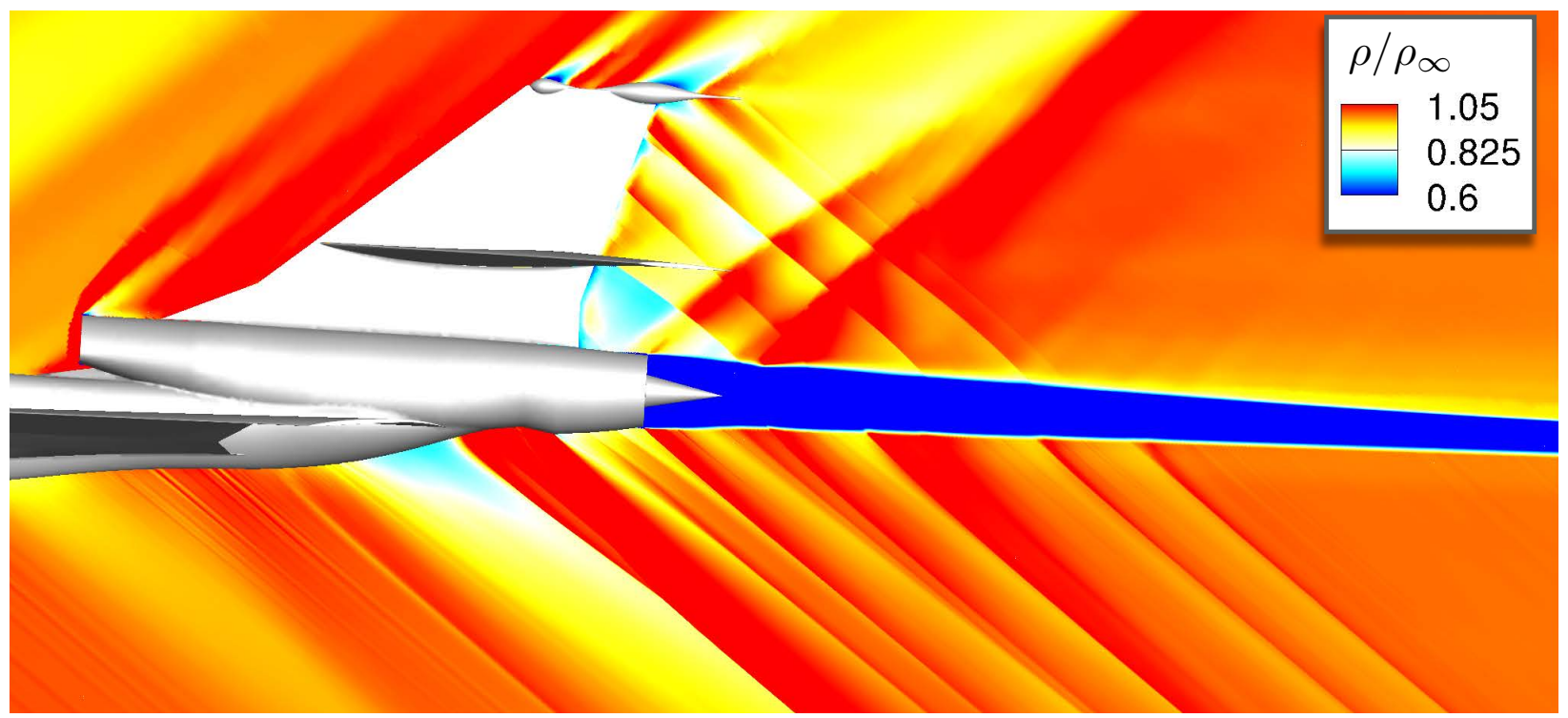

Figure 18: Density ratio contours around $\mathrm{C} 25 \mathrm{P}$ engine $\left(\Phi=0^{\circ}\right.$ mesh with $35 \mathrm{M}$ cells $)$

We use similar adaptation weights to the $\mathrm{C} 25 \mathrm{~F}$ case and a $w(\ell)$ profile similar to the JWB case (Figure 14), with a higher weighted nose and initial plume region. An ancillary pressure line sensor was also added along the plume core to encourage smoother refinement of the plume. We found keeping the mesh smooth in the plume to be challenging, but critical to obtaining a reasonable off-body signature. This is consistent with the workshop's suggested meshing guidelines for the plume region.

We performed mesh refinement investigations to identify meshing requirements and submitted a mesh sequence with on average about 6,12 , and $35 \mathrm{M}$ cells per azimuth. This is about $50 \%$ more than for the $\mathrm{C} 25 \mathrm{~F}$, which is in line with our experience with other powered boom problems. The presence of a plume tends to greatly increase meshing requirements.

Figure 19 shows the fine-mesh $r / L=5$ signatures at every azimuth. Interestingly, the aft portion of the final signatures are fairly unremarkable. This should not indicate the simplicity of the problem - coarser or uneven meshing at the plume boundaries tends to generate spurious artifacts in the flow solution. This is evident in the error estimates in Figure 19, which indicate that the mesh sequences generally exhibit poor asymptotic convergence. While we verified that the fine mesh solutions capture all the significant features of ultra-fine reference meshes, the coarser meshes submitted are not as convincing. 
$\mathrm{C} 25 \mathrm{P}\left(\mathrm{r} / \mathrm{L}=5, \Phi=0^{\circ}\right)$

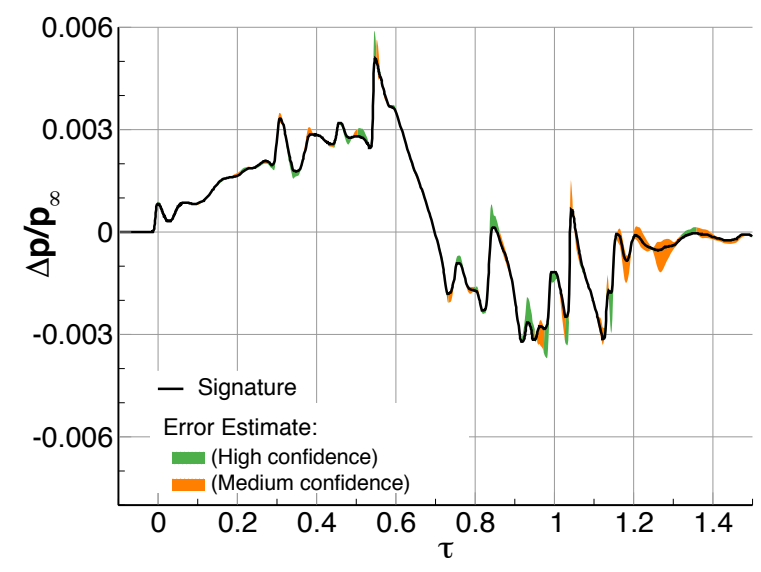

$\mathrm{C} 25 \mathrm{P}\left(\mathrm{r} / \mathrm{L}=5, \Phi=20^{\circ}\right)$

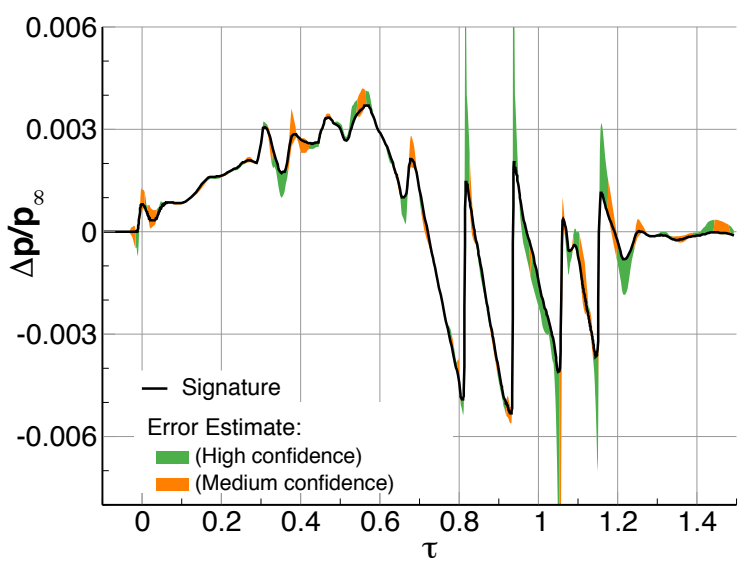

$\mathrm{C} 25 \mathrm{P}\left(\mathrm{r} / \mathrm{L}=5, \Phi=40^{\circ}\right)$

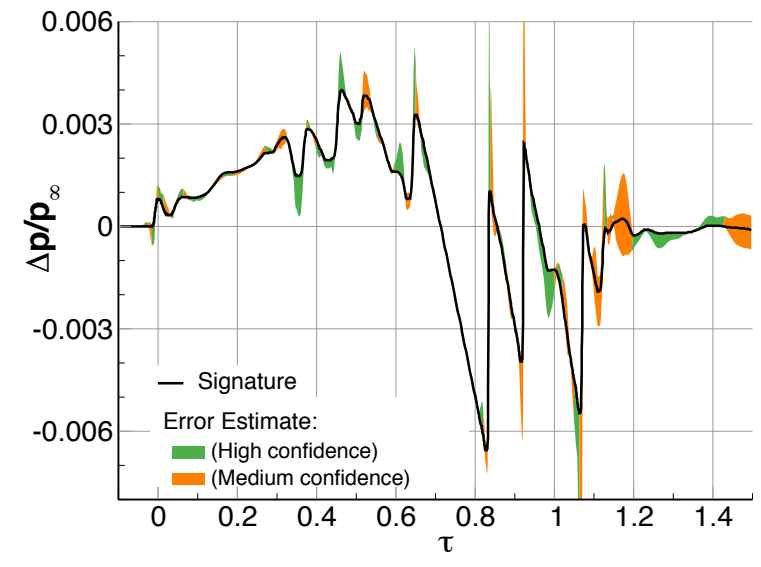

$\mathrm{C} 25 \mathrm{P}\left(\mathrm{r} / \mathrm{L}=5, \Phi=10^{\circ}\right)$

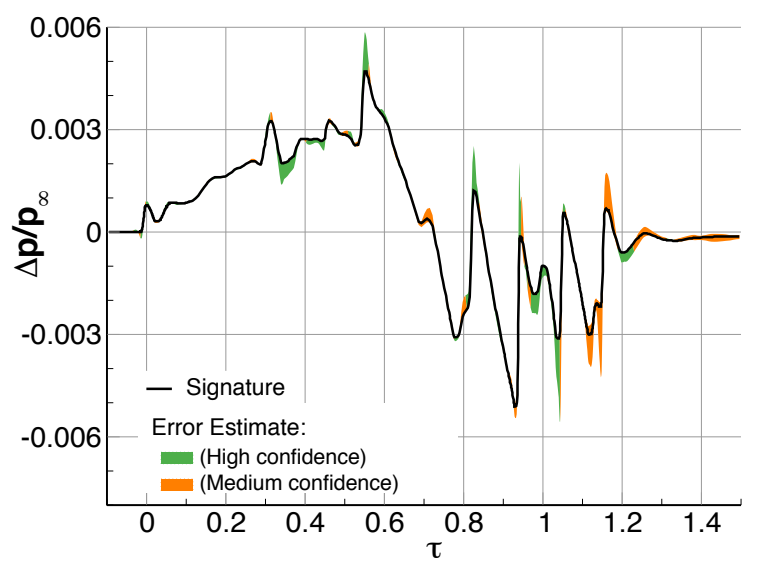

$\mathrm{C} 25 \mathrm{P}\left(\mathrm{r} / \mathrm{L}=5, \Phi=30^{\circ}\right)$

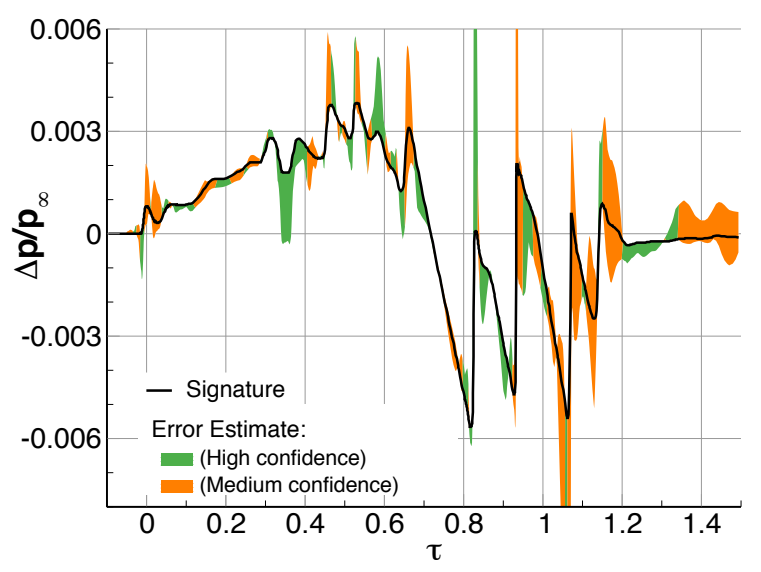

$\mathrm{C} 25 \mathrm{P}\left(\mathrm{r} / \mathrm{L}=5, \Phi=50^{\circ}\right)$

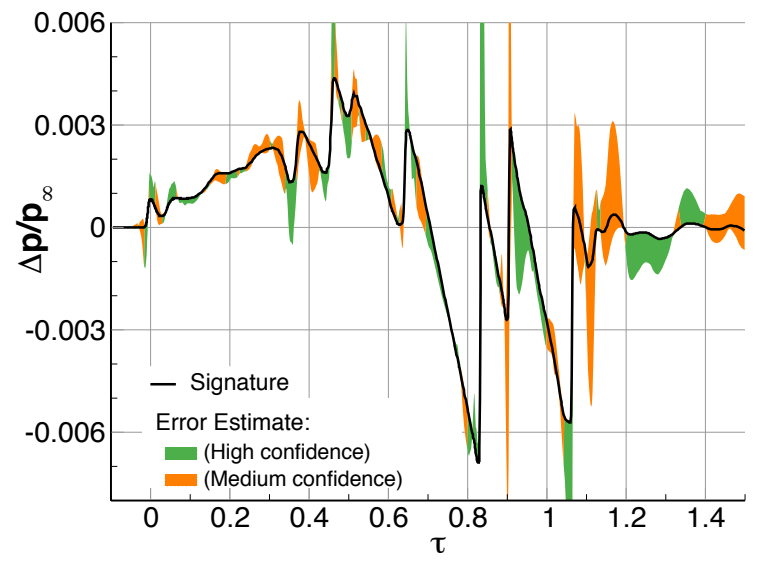

Figure 19: C25P: Pressure signature carpet at $r / L=5$, showing fine mesh signature and local error estimates $\left(E^{\mathrm{mf}}, E^{\mathrm{cm}}\right)$. $x$-axis shows $\tau=(x-r \beta) / L$. To stabilize error estimates, coarse submitted meshes were replaced by "very coarse" meshes at all but $\Phi=0^{\circ}$. 


\section{III.F. Propagation and Loudness of Nearfield Signatures}

For each nearfield case at each azimuth, we propagated the farthest off-body, fine-grid nearfield signatures through a standard atmosphere, using SBOOM. We applied best practices determined in the propagation portion of the workshop, which will be discussed in Section IV. The sampling frequency was set to $200 \mathrm{kHz}$ (about $40 \mathrm{~K}$ points), based on studies of mesh convergence with SBOOM. After propagation, the PL and ASEL noise metrics were computed using LCASB. Table 4 shows these metrics for all cases and azimuths. Interestingly, for all cases, the loudest predicted sonic booms are generally around $\Phi=30^{\circ}$, highlighting the importance of even moderately large off-track angles.

To investigate the sensitivity of noise metrics to the CFD mesh resolution, the propagation and loudness computations were repeated for nearfield signatures computed on the coarse and medium grids. Each PLdB noise value in Table 4 is accompanied by a number indicating the difference (in $\mathrm{dB}$ ) between the fine CFD grid and coarse-grid noise predictions. For all cases, at all azimuths, the maximum change in PL is $1.6 \mathrm{~dB}$ (and usually less than $1 \mathrm{~dB}$ ). However, we note that in most cases the noise metrics do not yet indicate asymptotic convergence, even on the JWB case, which we consider to be sufficiently resolved based on both the adjoint-based error estimates and the independent localized error estimates.

Table 4: Ground noise for all nearfield cases and azimuths, propagated from nearfield signatures at $r / L=5$ on fine CFD meshes. Bold: Loudest azimuth, $\boldsymbol{\nabla}$ and $\boldsymbol{\Delta}$ indicate change from the coarse CFD mesh.

\begin{tabular}{|c|c|c|c|c|c|c|c|}
\hline & \multirow[b]{2}{*}{ Case } & \multicolumn{6}{|c|}{ PLdB } \\
\hline & & $\Phi=0^{\circ}$ & $\Phi=10^{\circ}$ & $\Phi=20^{\circ}$ & $\Phi=30^{\circ}$ & $\Phi=40^{\circ}$ & $\Phi=50^{\circ}$ \\
\hline I. & AXIE & $78.1(\mathbf{v} 0.4)$ & - & - & - & - & - \\
\hline II. & JWB & $79.5(\nabla 0.6)$ & $76.5(\vee 0.7)$ & $78.2(\mathbf{v} 0.4)$ & $82.2(\nabla 1.5)$ & $81.6(\mathbf{\nabla} 0.1)$ & $76.6(\Delta 0.5)$ \\
\hline III. & $\mathrm{C} 25 \mathrm{~F}$ & $78.1(\mathbf{\Delta} 0.8)$ & $80.4(\boldsymbol{\Delta} 0.6)$ & $80.1(\mathbf{\nabla} 0.1)$ & $\mathbf{8 2 . 2}(\triangle 0.8)$ & $80.1(\boldsymbol{\Delta} 0.6)$ & $73.3(0.0)$ \\
\hline \multirow[t]{3}{*}{ IV. } & $\mathrm{C} 25 \mathrm{P}$ & $80.4(\mathbf{\nabla} 0.5)$ & $81.3(\boldsymbol{\nabla} 0.5)$ & $78.3(\mathbf{v} 0.3)$ & $\mathbf{8 1 . 4}(\vee 0.6)$ & $78.7(\mathbf{\nabla} 0.4)$ & $73.3(\mathbf{v} 1.6)$ \\
\hline & & \multicolumn{6}{|c|}{$\mathrm{dB}(\mathrm{A})$} \\
\hline & & $\Phi=0^{\circ}$ & $\Phi=10^{\circ}$ & $\Phi=20^{\circ}$ & $\Phi=30^{\circ}$ & $\Phi=40^{\circ}$ & $\Phi=50^{\circ}$ \\
\hline I. & AXIE & 63.7 & - & - & - & - & - \\
\hline II. & JWB & 65.4 & 62.7 & 64.4 & 67.6 & 67.2 & 61.8 \\
\hline III. & $\mathrm{C} 25 \mathrm{~F}$ & 63.5 & 66.3 & 65.9 & 67.8 & 65.4 & 58.7 \\
\hline IV. & $\mathrm{C} 25 \mathrm{P}$ & 66.2 & 67.4 & 63.7 & 66.9 & 64.4 & 58.6 \\
\hline
\end{tabular}

\section{III.G. Computational Cost}

The mesh sizes and computational resources used are summarized in Table 5 . The total computational cost for all the submitted CFD simulations was 1450 corehours. For each case, all azimuths are completely independent and were run in parallel on separate 28-core compute nodes. Thus, the total wall-clock time for each case was under five hours. This highlights one major advantage of azimuthal splitting, at least when high-capacity computing resources are available. Using a single mesh for each carpet would have taken far longer, both because the parallel scaling would not be as ideal, and also because we would not be able to take advantage of improved azimuthal mesh alignment and higher stretching. Roughly half of the total wall-clock time per case is spent on adaptive meshing (including all intermediate flow and adjoint solves and error estimation), and the other half on the fine-mesh flow solutions.
Table 5: Computational cost for nearfield cases. Time includes all meshing and adaptation. Each off-track angle of each case was solved on its own dual-socket computing node with 28 hardware cores (Intel Xeon E5-2680v4, "Broadwell") and $128 \mathrm{~GB}$ of memory.

\begin{tabular}{rlccc|cc}
\hline & \multicolumn{4}{c}{ Fine mesh cell counts } & Time & Core-hrs \\
& Case & per $\Phi^{*}$ & $N_{\Phi}$ & SBPw2 & per $\Phi^{*}$ & Total \\
\hline I. & AXIE & $26 \mathrm{M}$ & $\times 1$ & $56 \mathrm{M}$ & $1.3 \mathrm{~h}$ & 36 \\
II. & JWB & $32 \mathrm{M}$ & $\times 6$ & $18 \mathrm{M}$ & $2.5 \mathrm{~h}$ & 414 \\
III. & C25F & $19 \mathrm{M}$ & $\times 6$ & $104 M$ & $1.5 \mathrm{~h}$ & 252 \\
IV. & C25P & $35 \mathrm{M}$ & $\times 6$ & $52 M$ & $4.5 \mathrm{~h}$ & 748 \\
\hline
\end{tabular}

${ }^{*}$ Average across all $\Phi$, on finest submitted grids

$\dagger$ Control volume count of the finest unstructured, tetrahedral inviscid grid provided by the workshop 


\section{Propagation Results}

We solve both of the propagation cases, at all azimuths and for all required and optional atmospheric conditions. For each case, nearfield pressure signature carpets are prescribed, and participants propagate these signals to the ground through various prescribed atmospheric conditions, some of which include non-standard temperature, pressure, humidity and wind profiles.

The first case involves a signature derived from the axisymmetric geometry used as Case I (AXIE) in the nearfield portion of the workshop (see Section III.B). For clarity, we will refer to this propagation case as AXIE-PROP. The second test case corresponds to the LM-1021 low-boom aircraft example that was used in SBPW1. ${ }^{2,24}$ In order to ensure that all participants started from a common baseline, these prescribed nearfield signatures are completely independent of the participants' own signatures submitted in SBPW1 or SBPW2.

As before, SBOOM (v2.5) ${ }^{19}$ is used for propagation and noise metrics are computed with LCASB. ${ }^{20}$ SBOOM solves a quasi-one- Table 6: sBOOM sampling frequencies dimensional PDE via finite difference and is therefore subject to truncation error. The accuracy of the propagated signal is therefore directly influenced by the mesh spacing along the waveform. This spacing is expressed as a sampling frequency (points per second of the waveform). Mesh convergence studies (spanning $35-500 \mathrm{kHz}$ ) were conducted for both cases to determine the required resolution determined by mesh refinement studies

\begin{tabular}{rlc}
\hline & Case & Samp. Freq. \\
\hline I. & AXIE-PROP & $107 \mathrm{kHz}$ \\
II. & LM-1021 & $75.6 \mathrm{kHz}$ \\
\hline
\end{tabular}
to converge ASEL to within $\pm 0.075 \mathrm{~dB}$ and PL to within $\pm 0.1 \mathrm{~dB}$. The resulting sampling frequencies are given in Table 6 . Although these frequencies are quite different, they actually represent a similar number of points (here $\sim 30 \mathrm{~K}$ points), due to the different signal lengths. The provided nearfield signatures are coarser by a factor of $10-100 \times(\sim 1725$ points for AXIE-PROP and $\sim 200$ points per azimuth for LM-1021). This implies that significant oversampling of the nearfield signal is required to control discretization error during signal propagation. We experimented with splining the nearfield signatures but ultimately chose to use the default linear interpolation in SBOOM - this remains an open question.

\section{IV.A. Case I: AXIE-PROP - Axisymmetric Body for Propagation}

The test conditions for the AXIE-PROP case correspond to flight at Mach 1.6 at an altitude of $15.85 \mathrm{~km}$. The provided nearfield signal is located at $r / L=3$, with the reference length $L=42.98 \mathrm{~m}$. Propagation was requested for three azimuths, $\Phi=\left\{-45^{\circ}, 0^{\circ}, 45^{\circ}\right\}$. Figure 20 shows the computed ground signatures for these azimuths through the four prescribed atmospheric profiles. The first two are based on the standard atmosphere, ${ }^{25}$ which has no wind:

Std. Atm. (Top Left) The standard atmosphere.

Std. Atm.+70\% Humidity (Bottom Right) The standard atmosphere, but with uniform $70 \%$ humidity at all altitudes.

Since there is no wind in these two profiles, the propagated signals at $\pm 45^{\circ}$ overplot in Figure 20 . The other two atmospheres include wind:

Atm. \#3 (Top Right) Data taken on a hot day near Wallops Island, Maryland.

Atm. \#4 (Bottom Left) Data taken on a hot, dry day in August near Edwards Air Force Base, California.

Compared to the standard atmosphere, these two were expected to produce louder and quieter noise metrics, respectively. The results in the corresponding frames of Figure 20 are consistent with these expectations: Atm. \#3 has the highest peak-to-peak signal $\left(\right.$ at $\Phi=0^{\circ}$ ), while Atm. \#4 has the lowest. In fact, the hot dry air in Atm. \#4 dampens the signal so much that, with the prevailing wind profile, the ray tube corresponding to $\Phi=-45^{\circ}$ never reaches the ground, being beyond the signal cutoff angle of $-43.9^{\circ}$.

Table 7 contains values of perceived level of noise (PLdB), and A- and C-weighted sound exposure levels $(\mathrm{dB}(\mathrm{A})$ and $\mathrm{dB}(\mathrm{C}))$ for all three azimuths through each of the four atmospheres. Signal cutoff angles were computed for each atmosphere using SBOOM's ray tube method. ${ }^{\text {f }}$ These cutoff angles are listed in Table 8, along with the ground location of the limiting rays (relative to the aircraft) and the total track width of the ground carpet.

\footnotetext{
${ }^{\mathrm{f}}$ We ran SBOOM (v2.5) iteratively within a binary-search driver. Newer versions of SBOOM have since added this capability, though with a different search method.
} 

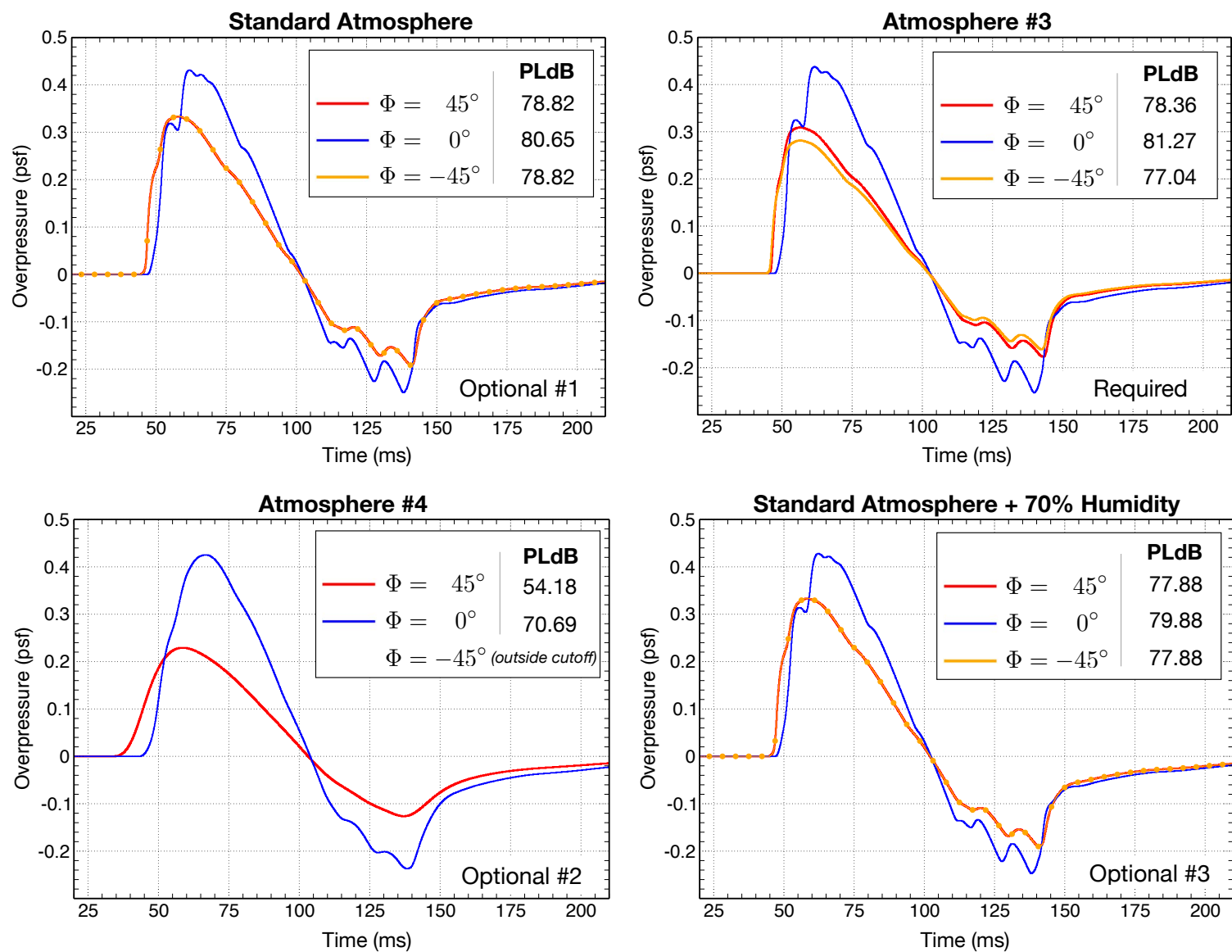

Figure 20: AxIE-PROP: Ground signatures at $\Phi=\left\{-45^{\circ}, 0^{\circ}, 45^{\circ}\right\}$ for all atmospheric profiles

\begin{tabular}{c|c|c|c|c|c|c|c|c|c} 
& \multicolumn{3}{|c|}{ PLdB } & \multicolumn{3}{c|}{ dB(A) } & \multicolumn{3}{c}{ dB(C) } \\
\hline $\begin{array}{c}\text { Atmosphere } \\
\text { Profile }\end{array}$ & $-45^{\circ}$ & $0^{\circ}$ & $+45^{\circ}$ & $-45^{\circ}$ & $0^{\circ}$ & $+45^{\circ}$ & $-45^{\circ}$ & $0^{\circ}$ & $+45^{\circ}$ \\
\hline Std. Atm. & 78.82 & 80.65 & 78.82 & 64.40 & 66.06 & 64.40 & 90.09 & 91.90 & 90.09 \\
\hline $\begin{array}{c}\text { Std. Atm. + } \\
\text { 70\% humidity }\end{array}$ & 77.88 & 79.88 & 77.88 & 63.30 & 65.30 & 63.30 & 89.97 & 91.75 & 89.97 \\
\hline Atm. \# 3 & 77.04 & 81.27 & 78.36 & 62.39 & 66.86 & 63.66 & 88.74 & 92.15 & 89.65 \\
\hline Atm. \# 4 & $\begin{array}{c}\text { Beyond } \\
\text { cutoff }\end{array}$ & 70.69 & 54.18 & $\begin{array}{c}\text { Beyond } \\
\text { cutoff }\end{array}$ & 55.87 & 44.46 & $\begin{array}{c}\text { Beyond } \\
\text { cutoff }\end{array}$ & 90.54 & 84.49 \\
\hline
\end{tabular}

Table 7: AxIE-Prop: Noise metrics (PLdB, $\mathrm{dB}(\mathrm{A}), \mathrm{dB}(\mathrm{C}))$ at $\Phi=\left\{-45^{\circ}, 0^{\circ}, 45^{\circ}\right\}$ for all atmospheric profiles

\begin{tabular}{cccc}
$\begin{array}{c}\text { Atmosphere } \\
\text { Profile }\end{array}$ & $\begin{array}{c}\text { Cutoff }\left(-\phi^{\circ}\right) \\
(\mathrm{x}, \mathrm{y}) \mathrm{km}\end{array}$ & $\begin{array}{c}\text { Cutoff }\left(+\phi^{\circ}\right) \\
(\mathrm{x}, \mathrm{y}) \mathrm{km}\end{array}$ & Track Width \\
\hline Std. Atm. & $\begin{array}{c}-50.4^{\circ} \\
(35.9,-34.5) \mathrm{km}\end{array}$ & $\begin{array}{c}50.4^{\circ} \\
(35.9,34.5) \mathrm{km}\end{array}$ & $69.0 \mathrm{~km}$ \\
\hline $\begin{array}{c}\text { Std. Atm. }+ \\
\text { 70\% humidity }\end{array}$ & $\begin{array}{c}-50.4^{\circ} \\
(35.9,-34.5) \mathrm{km}\end{array}$ & $\begin{array}{c}50.4^{\circ} \\
(35.9,34.5) \mathrm{km}\end{array}$ & $69.0 \mathrm{~km}$ \\
\hline Atm. \# 3 & $\begin{array}{c}-53.1^{\circ} \\
(48.5,-46.1) \mathrm{km}\end{array}$ & $\begin{array}{c}50.3^{\circ} \\
(44.1,39.3) \mathrm{km}\end{array}$ & $85.4 \mathrm{~km}$ \\
\hline Atm. \# 4 & $\begin{array}{c}-43.9^{\circ} \\
(35.9,-30.7) \mathrm{km}\end{array}$ & $\begin{array}{c}46.7^{\circ} \\
(44.9,40.8) \mathrm{km}\end{array}$ & $71.5 \mathrm{~km}$ \\
\hline
\end{tabular}

Table 8: AxiE-Prop: Raytube cutoff angles and ground intersection location of limiting ray for all atmospheric profiles. "Track Width" denotes the component of the ground distance between the two limiting rays normal to the flight path. 

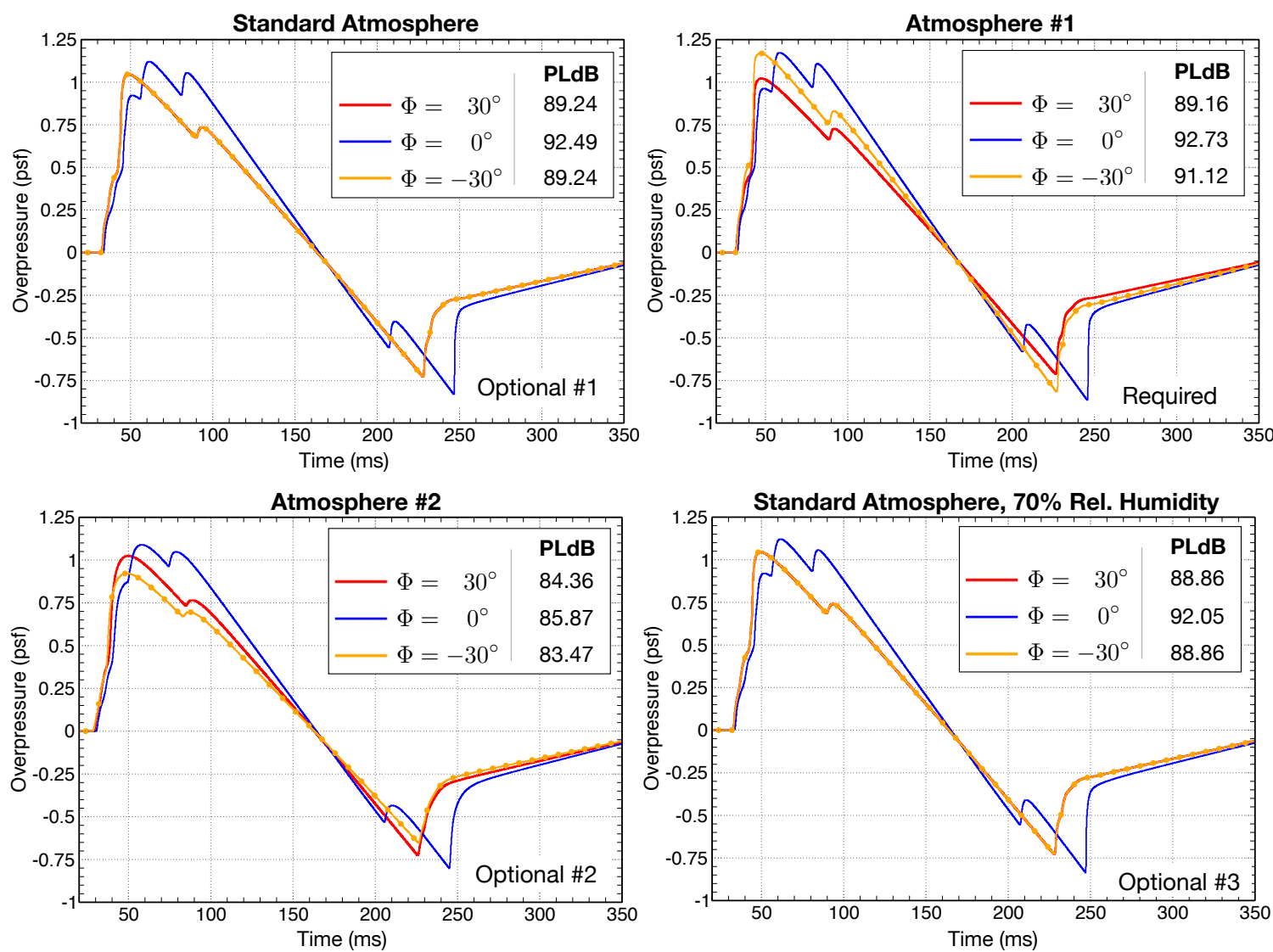

Figure 21: LM-1021: Ground signatures at $\Phi=\left\{-30^{\circ}, 0^{\circ}, 30^{\circ}\right\}$ for all atmospheric profiles

\begin{tabular}{c|c|c|c|c|c|c|c|c|c} 
& \multicolumn{3}{|c|}{ PLdB } & \multicolumn{3}{c|}{ dB(A) } & \multicolumn{3}{c}{ dB(C) } \\
\hline $\begin{array}{c}\text { Atmosphere } \\
\text { Profile }\end{array}$ & $-30^{\circ}$ & $0^{\circ}$ & $+30^{\circ}$ & $-30^{\circ}$ & $0^{\circ}$ & $+30^{\circ}$ & $-30^{\circ}$ & $0^{\circ}$ & $+30^{\circ}$ \\
\hline $\begin{array}{c}\text { Std. Atm. } \\
\text { Std. Atm. + }\end{array}$ & 89.24 & 92.49 & 89.24 & 74.19 & 78.08 & 74.19 & 97.87 & 97.92 & 97.87 \\
\hline $\begin{array}{c}\text { 70\% humidity } \\
\text { Atm. \# 1 }\end{array}$ & 98.86 & 92.05 & 88.86 & 73.68 & 77.50 & 73.68 & 97.79 & 97.82 & 97.79 \\
\hline Atm. \# 2 & 83.47 & 85.87 & 84.36 & 68.39 & 70.77 & 69.07 & 96.64 & 96.85 & 97.37 \\
\hline
\end{tabular}

Table 9: LM-1021: Noise metrics (PLdB, $\mathrm{dB}(\mathrm{A}), \mathrm{dB}(\mathrm{C}))$ at $\Phi=\left\{-30^{\circ}, 0^{\circ}, 30^{\circ}\right\}$ for all atmospheric profiles

\begin{tabular}{cccc}
$\begin{array}{c}\text { Atmosphere } \\
\text { Profile }\end{array}$ & $\begin{array}{c}\text { Cutoff }\left(-\phi^{\circ}\right) \\
(x, y) \mathrm{km}\end{array}$ & $\begin{array}{c}\text { Cutoff }\left(+\phi^{\circ}\right) \\
(x, y) \mathrm{km}\end{array}$ & Track Width \\
\hline Std. Atm. & $\begin{array}{c}-50.4^{\circ} \\
(37.0,-35.6) \mathrm{km}\end{array}$ & $\begin{array}{c}50.4^{\circ} \\
(37.0,35.6) \mathrm{km}\end{array}$ & $71.2 \mathrm{~km}$ \\
\hline $\begin{array}{c}\text { Std. Atm. }+ \\
\text { 70\% humidity }\end{array}$ & $\begin{array}{c}-50.4^{\circ} \\
(37.0,-35.6) \mathrm{km}\end{array}$ & $\begin{array}{c}50.4^{\circ} \\
(37.0,35.6) \mathrm{km}\end{array}$ & $71.2 \mathrm{~km}$ \\
\hline Atm. \# 1 & $\begin{array}{c}-74.0^{\circ} \\
(39.4,-44.6) \mathrm{km}\end{array}$ & $\begin{array}{c}57.5^{\circ} \\
(40.0,42.3) \mathrm{km}\end{array}$ & $86.9 \mathrm{~km}$ \\
\hline Atm. \# 2 & $\begin{array}{c}-59.4^{\circ} \\
(67.0,-69.7) \mathrm{km}\end{array}$ & $\begin{array}{c}64.7^{\circ} \\
(43.9,41.7) \mathrm{km}\end{array}$ & $111.4 \mathrm{~km}$ \\
\hline
\end{tabular}

Table 10: LM-1021: Raytube cutoff angles and ground intersection location of limiting ray for all atmospheric profiles. "Track Width" denotes the component of the ground distance between the two limiting rays normal to the flight path. 


\section{IV.B. Case II: LM-1021}

Flight conditions for the LM-1021 correspond to a cruise Mach number of 1.6 at $16.76 \mathrm{~km}$ altitude. The nearfield signatures were collected at $r=222.6 \mathrm{~m}$ from the aircraft flight path $(r / L=3.13)$. Pressure distributions were provided at $\Phi=\left\{-30^{\circ}, 0^{\circ}, 30^{\circ}\right\}$. Figure 21 shows the computed ground signatures for these azimuths through all four atmospheric profiles. As before, the top left frame corresponds to the standard atmosphere, while the bottom right frame is for the the standard atmosphere with $70 \%$ humidity. Again, in these quiescent atmospheres, ground signatures for the $\pm \Phi$ azimuths overplot. The other two atmospheres include wind, and are based on sounding data collected near Green Bay, Wisconsin on two consecutive winter days with unsettled weather:

Atm. \#1 (Top Right) Day 1 (expected to be louder)

Atm. \#2 (Bottom Left) Day 2 (expected to be quieter)

The significant disparity between the ground profiles at $\Phi= \pm 30^{\circ}$ in Figure 21 for these two atmospheres is indicative of significant winds aloft in each profile. Table 9 provides all computed noise metrics at each azimuth, for each atmosphere. As expected, Atms. \#1 and \#2 are louder and quieter, respectively. The importance of wind is apparent in Atm. $\# 1$, especially at $\Phi=-30^{\circ}$, where the ground signal is almost 2 PLdB louder than at the same azimuth in the standard atmosphere. In the CSEL metric, $\Phi=-30^{\circ}$ is even louder than the on-track signal by about $0.75 \mathrm{~dB}$.

Table 10 lists the signal cutoff angles for each of the atmospheric profiles. Examining the cold and windy Atm. \#1, the computed $-\Phi$ cutoff is $-74^{\circ}$. This is significantly greater than the cutoff angles of $45-55^{\circ}$ typically seen without wind. Near the limiting azimuth, the ray tube analysis shows that the sound took over five minutes to reach the ground - over three times longer than without wind. Atm. \#2 is noteworthy as well. On-track it is over $6.5 \mathrm{~dB}$ quieter than the standard atmosphere in both PL and ASEL. Moreover, the track width of the ground carpet is over $50 \mathrm{~km}$ broader than for the still air of the standard atmosphere.

\section{Summary and Discussion}

As with previous workshops, participation in the nearfield portion of SBPW2 directly drove major meshing efficiency improvements for computing off-body signatures with Cartesian meshes. The largest gains were obtained by fully embracing a parallel mesh-splitting approach, where each off-track angle of the discretized boom carpet is computed on its own azimuthally-aligned mesh. We further reduced cell counts by using more aggressive aspect ratios than in previous work (up to 8). As in previous studies, adjoint-based mesh adaptation proved highly effective at focusing the meshing effort. Unlike in previous work, where dozens of signatures were computed in a monolothic mesh, here each mesh handles at most three signatures. This also simplified the signature weighting.

As in previous workshops, the aft part of each signature remains the most challenging to compute reliably and affordably. Wake and plume regions exhibit strong mesh-sensitivity (see especially the JWB case results) and require much higher resolution. To help visualize this sensitivity, we introduced a method for localizing discretization error, based on Richardson extrapolation of signatures computed on a sequence of three meshes. This provides an informative, independent verification of both manually refined meshes (such as the workshop-provided grids) and meshes constructed via adaptation.

The introduction of atmospheric propagation to the workshop provided a welcome, practical focus to the interpretation of mesh convergence of CFD results. In several of the cases, we showed that mesh sequences that exhibit convergent behavior in a CFD-oriented metric (e.g. an adaptation functional) do not necessarily exhibit asymptotic behavior in terms of ground loudness. On the other hand, the computed noise metrics are remarkably stable, varying by less than about $1.5 \mathrm{~dB}$ in PL across the three finest CFD grids. It is difficult to draw more rigorous conclusions about mesh convergence without a much deeper understanding of the interplay among CFD tools, propagation codes, and loudness metrics.

In the propagation portion of the workshop, the atmospheric profile was found to impact the ground loudness by as much as $8 \mathrm{~dB}$. In the presence of wind, we observed surprisingly extreme cutoff angles (as high as $74^{\circ}$ ) and track widths (up to $110 \mathrm{~km}$ ). Moreover, all of the nearfield vehicle configurations were found to generate louder $30^{\circ}$ off-track booms than the on-track boom. These observations strongly justify the increasing focus on computing moderately wide boom carpets and incorporating the effects of atmospheric variability. 


\section{Acknowledgments}

The authors sincerely thank the members of the SBPw2 organizing committee for their diligence and responsiveness throughout this effort, especially Mike Park (Langley) and also Sriram Rallabhandi (Langley), who assisted with questions regarding SBOOM. We are grateful to Mathias Wintzer (Gulfstream) and David Rodriguez (NASA Ames) for helpful discussions and for sharing their insight into supersonic flight and boom prediction. This research was supported by the NASA ARMD Commercial Supersonic Technology (CST) Project and by NASA Ames Research Center contract NNA16BD60C.

\section{References}

${ }^{1}$ Park, M. A., Aftosmis, M. J., Campbell, R. L., Carter, M. B., Cliff, S. E., and Bangert, L. S., "Summary of the 2008 NASA Fundamental Aeronautics Program Sonic Boom Prediction Workshop," Journal of Aircraft, Vol. 51, No. 3, 2017/04/28 2014, pp. 987-1001.

${ }^{2}$ Park, M. A. and Morgenstern, J. M., "Summary and Statistical Analysis of the First AIAA Sonic Boom Prediction Workshop," 32nd AIAA Applied Aerodynamics Conference, Atlanta, GA, June 2014.

${ }^{3}$ Park, M. A. and Nemec, M., "Nearfield Summary and Statistical Analysis of the Second AIAA Sonic Boom Prediction Workshop," 35th AIAA Applied Aerodynamics Conference, 2017.

${ }^{4}$ Wintzer, M., Nemec, M., and Aftosmis, M. J., "Adjoint-Based Adaptive Mesh Refinement for Sonic Boom Prediction," 26th AIAA Applied Aerodynamics Conference, August 2008.

${ }^{5}$ Aftosmis, M. J., Nemec, M., and Cliff, S. E., Adjoint-Based Low-Boom Design with Cart3D, 29th AIAA Applied Aerodynamics Conference, Honolulu, HI, June 2011.

${ }^{6}$ Aftosmis, M. J. and Nemec, M., Cart3D Simulations for the First AIAA Sonic Boom Prediction Workshop, 52nd Aerospace Sciences Meeting, January 2014.

${ }^{7}$ Lomax, H., "The Wave Drag Of Arbitrary Configurations In Linearized Flow As Determined By Areas And Forces In Oblique Planes," Research memorandum, Ames Aeronautical Laboratory, Washington, D.C., 1955.

${ }^{8}$ Aftosmis, M. J., Berger, M. J., and Adomavicius, G., A Parallel Multilevel Method for Adaptively Refined Cartesian Grids with Embedded Boundaries, American Institute of Aeronautics and Astronautics, Reno, NV, January 2000.

${ }^{9}$ Berger, M. J., Aftosmis, M. J., Marshall, D. D., and Murman, S. M., "Performance of a New CFD Flow Solver Using a Hybrid Programming Paradigm," J. Parallel Distrib. Comput., Vol. 65, No. 4, April 2005, pp. 414-423.

${ }^{10}$ van Leer, B., Flux-vector splitting for the Euler equations, Springer Berlin Heidelberg, Berlin, Heidelberg, 1982, pp. $507-512$.

${ }^{11}$ Barth, T. and Jespersen, D., The design and application of upwind schemes on unstructured meshes, American Institute of Aeronautics and Astronautics, 2017/05/08 1989.

${ }^{12}$ Wintzer, M. and Ordaz, I., "Under-Track CFD-Based Shape Optimization for a Low-Boom Demonstrator Concept," 33rd AIAA Applied Aerodynamics Conference, 2015.

${ }^{13}$ Aftosmis, M. J., Berger, M. J., and Melton, J. E., "Robust and Efficient Cartesian Mesh Generation for Component-Based Geometry," AIAA Journal, Vol. 36, No. 6, June 1998.

${ }^{14}$ Nemec, M., Aftosmis, M. J., Murman, S. M., and Pulliam, T. H., "Adjoint Formulation for an Embedded-Boundary Cartesian Method," 43rd AIAA Aerospace Sciences Meeting, Reno, NV, January 2005.

${ }^{15}$ Haering, E., Murray, J., Purifoy, D., Graham, D., Meredith, K., Ashburn, C., and Stucky, M., Airborne Shaped Sonic Boom Demonstration Pressure Measurements with Computational Fluid Dynamics Comparisons, American Institute of Aeronautics and Astronautics, 2017/05/01 2005.

${ }^{16}$ Moschetta, J.-M. and Gressier, J., The Sonic Point Glitch Problem: A Numerical Solution, Springer Berlin Heidelberg, Berlin, Heidelberg, 1998, pp. 403-408.

${ }^{17}$ Nemec, M. and Aftosmis, M. J., "Toward Automatic Verification of Goal-Oriented Flow Simulations," Tech. Memorandum TM-2014-218386, NASA, 2014.

${ }^{18}$ Roache, P. J., "Verification of Codes and Calculations," AIAA Journal, Vol. 36, No. 5, May 1998, pp. 696-702.

${ }^{19}$ Rallabhandi, S. K., "Advanced Sonic Boom Prediction Using the Augmented Burgers Equation," Journal of Aircraft, Vol. 48, No. 4, 2011, pp. 1245-1253.

${ }^{20}$ Kevin P., S. and Brenda M., S., "A Loudness Calculation Procedure Applied to Shaped Sonic Booms," Technical Paper 3134, NASA Langley, 1991.

${ }^{21}$ Stevens, S. S., "Perceived Level of Noise by Mark VII and Decibels (E)," The Journal of the Acoustical Society of America, Vol. 51, No. 2B, February 1972, pp. 575-601.

${ }^{22}$ Pratt, M. J., "Introduction to ISO 10303 - the STEP Standard for Product Data Exchange," J. Comput. Info. Sci. Eng., Vol. 1, No. 1, March 2001, pp. 102-103.

${ }^{23}$ Ueno, A., Kanamori, M., and Makino, Y., Multi-fidelity Low-boom Design Based on Near-field Pressure Signature, American Institute of Aeronautics and Astronautics, 2017/05/03 2016.

${ }^{24}$ Morgenstern, J. M., Buonanno, M., and Marconi, F., Full Configuration Low Boom Model and Grids for 2014 Sonic Boom Prediction Workshop, 51st AIAA Aerospace Sciences Meeting, Grapevine, TX, Jan. 2013.

${ }^{25}$ Internat. Org. for Standardization and U. S. Environmental Protection Agency, Standard Atmosphere: ISO 2533:1975, International Organization for Standardization, December 1978. 